Article

\title{
Towards an Operational SAR-Based Rice Monitoring System in Asia: Examples from 13 Demonstration Sites across Asia in the RIICE Project
}

\author{
Andrew Nelson ${ }^{1, *}$, Tri Setiyono ${ }^{1, *}$, Arnel B. Rala ${ }^{1}$, Emma D. Quicho ${ }^{1}$, Jeny V. Raviz ${ }^{1}$, \\ Prosperidad J. Abonete ${ }^{1}$, Aileen A. Maunahan ${ }^{1}$, Cornelia A. Garcia ${ }^{1}$, Hannah Zarah M. Bhatti ${ }^{1}$, \\ Lorena S. Villano ${ }^{1}$, Pongmanee Thongbai ${ }^{1}$, Francesco Holecz ${ }^{2}$, Massimo Barbieri ${ }^{2}$, \\ Francesco Collivignarelli ${ }^{2}$, Luca Gatti ${ }^{2}$, Eduardo Jimmy P. Quilang ${ }^{3}$, Mary Rose O. Mabalay ${ }^{3}$, \\ Pristine E. Mabalot ${ }^{3}$, Mabel I. Barroga ${ }^{3}$, Alfie P. Bacong ${ }^{3}$, Norlyn T. Detoito ${ }^{3}$, \\ Glorie Belle Berja ${ }^{3}$, Frenciso Varquez ${ }^{3}$, Wahyunto ${ }^{4}$, Dwi Kuntjoro ${ }^{4}$, Sri Retno Murdiyati ${ }^{4}$, \\ Sellaperumal Pazhanivelan ${ }^{5}$, Pandian Kannan ${ }^{5}$, Petchimuthu Christy Nirmala Mary ${ }^{5}$, \\ Elangovan Subramanian ${ }^{5}$, Preesan Rakwatin ${ }^{6}$, Amornrat Intrman ${ }^{7}$, Thana Setapayak ${ }^{7}$, \\ Sommai Lertna ${ }^{7}$, Vo Quang Minh ${ }^{8}$, Vo Quoc Tuan ${ }^{8}$, Trinh Hoang Duong ${ }^{9}$, \\ Nguyen Huu Quyen ${ }^{\text {, }}$ Duong Van Kham ${ }^{9}$, Sarith Hin ${ }^{10}$, Touch Veasna ${ }^{10}$, Manoj Yadav ${ }^{11}$, \\ Chharom Chin ${ }^{12}$ and Nguyen Hong Ninh ${ }^{13}$
}

1 International Rice Research Institute (IRRI), Los Baños 4031, Philippines; E-Mails: a.rala@irri.org (A.B.R.); e.quicho@irri.org (E.D.Q.); j.raviz@irri.org (J.V.R.); p.abonete@irri.org (P.J.A.); a.maunahan@irri.org (A.A.M.); cornelia.garcia@irri.org (C.A.G.); h.bhatti@irri.org (H.Z.M.B.); 1.villano@irri.org (L.S.V.); p.thongbai@irri.org (P.T.)

2 Sarmap, Purasca 6989, Switzerland; E-Mails: fholecz@sarmap.ch (F.H.); mbarbieri@sarmap.ch (M.B.); fcolli@sarmap.ch (F.C.); lgatti@sarmap.ch (L.G.)

3 Philippine Rice Research Institute (PhilRice), Muñoz 3119, Philippines; E-Mails: ejp.quilang@philrice.gov.ph (E.J.P.Q.); mro.mabalay@philrice.gov.ph (M.R.O.M.); pe.mabalot@philrice.gov.ph (P.E.M.); mi.barroga@philrice.gov.ph (M.I.B.); alfiebacong@yahoo.com.ph (A.P.B.); norlyndetoito@gmail.com (N.T.D.); bgloriebelle@gmail.com (G.B.B.); flvarquez@yahoo.com (F.V.) Indonesian Center for Agricultural Land Resources Research and Development (ICALRD), Bogor 16123, Indonesia; E-Mails: wahyunto_wt@yahoo.co.id (W.); dwi_bgr@yahoo.com (D.K.); menik_bogor@yahoo.com (S.R.M.)

5 Directorate of Crop Management, Tamil Nadu Agricultural University (TNAU), Coimbatore 641003, India; E-Mails: pazhanivelans@gmail.com (S.P.); pandian.kannan@gmail.com (P.K.); chrismary@rediffmail.com (P.C.N.M.); selvisubbug@yahoo.co.in (E.S.)

6 Geo-Informatics and Space Technology Development Agency (GISTDA), Bangkok 10210, Thailand; E-Mail: preesan@gistda.or.th 
7 Thailand Rice Department (TRD), Bangkok 10400, Thailand;

E-Mails: paquitopookie@yahoo.com (A.I.); zasoodting@gmail.com (T.S.); sommai@brrd.mail.go.th (S.L.)

8 Department of Land Resources, Can Tho University (CTU), Can Tho 92000, Vietnam; E-Mails: vqminh@ctu.edu.vn (V.Q.M.); vqtuan@ctu.edu.vn (V.Q.T.)

9 Institute of Meteorology, Hydrology and Environment (IMHEN), Hanoi 100000, Vietnam; E-Mails: duong.trinhhoang@imh.ac.vn (T.H.D.); quyen.nguyenhuu@imh.ac.vn (N.H.Q.); Kham.duongvan@imh.ac.vn (D.V.K.)

${ }^{10}$ Cambodian Agricultural Research and Development Institute (CARDI), Phnom Penh 12413, Cambodia; E-Mails: sarith.hin@gmail.com (S.H.); tveasna@cardi.org.kh (T.V.)

11 Deutsche Gesellschaft für Internationale Zusammenarbeit (GIZ) GmbH, New Delhi 110029, India; E-Mail: manoj.yadav@giz.de

12 Swiss Agency for Development and Cooperation (SDC)-Cambodia, Phnom Penh 12413, Cambodia; E-Mail: chharom@gmail.com

13 Swiss Agency for Development and Cooperation (SDC)-Vietnam, Hanoi 100000, Vietnam; E-Mail: ninh.nguyen@eda.admin.ch

* Authors to whom correspondence should be addressed; E-Mails: a.nelson@irri.org (A.N.); t.setiyono@irri.org (T.S.); Tel.: +63-2-580-5600 (ext. 2592) (A.N.).

External Editors: Bingfang Wu, Nicolas Baghdadi and Prasad S. Thenkabail

Received: 30 June 2014; in revised form: 3 October 2014 / Accepted: 28 October 2014 / Published: 6 November 2014

\begin{abstract}
Rice is the most important food security crop in Asia. Information on its seasonal extent forms part of the national accounting of many Asian countries. Synthetic Aperture Radar (SAR) imagery is highly suitable for detecting lowland rice, especially in tropical and subtropical regions, where pervasive cloud cover in the rainy seasons precludes the use of optical imagery. Here, we present a simple, robust, rule-based classification for mapping rice area with regularly acquired, multi-temporal, X-band, HH-polarized SAR imagery and site-specific parameters for classification. The rules for rice detection are based on the well-studied temporal signature of rice from SAR backscatter and its relationship with crop stages. We also present a procedure for estimating the parameters based on "temporal feature descriptors" that concisely characterize the key information in the rice signatures in monitored field locations within each site. We demonstrate the robustness of the approach on a very large dataset. A total of 127 images across 13 footprints in six countries in Asia were obtained between October 2012, and April 2014, covering $4.78 \mathrm{~m}$ ha. More than 1900 in-season site visits were conducted across 228 monitoring locations in the footprints for classification purposes, and more than 1300 field observations were made for accuracy assessment. Some $1.6 \mathrm{~m}$ ha of rice were mapped with classification accuracies from $85 \%$ to $95 \%$ based on the parameters that were closely related to the observed temporal feature descriptors derived for
\end{abstract}


each site. The 13 sites capture much of the diversity in water management, crop establishment and maturity in South and Southeast Asia. The study demonstrates the feasibility of rice detection at the national scale using multi-temporal SAR imagery with robust classification methods and parameters that are based on the knowledge of the temporal dynamics of the rice crop. We highlight the need for the development of an open-access library of temporal signatures, further investigation into temporal feature descriptors and better ancillary data to reduce the risk of misclassification with surfaces that have temporal backscatter dynamics similar to those of rice. We conclude with observations on the need to define appropriate SAR acquisition plans to support policies and decisions related to food security.

Keywords: rice; food security; SAR; Asia; COSMO Skymed; TerraSAR-X

\section{Introduction}

\subsection{The Case for Synthetic Aperture Radar to Map Rice}

Rice is the most important crop for food security in Asia [1]. Despite rapid urbanization and diversification in consumption patterns in Asian countries, rice still accounts for $31 \%$ of the calorific intake and is by far the largest single source of calories for more than 3.7 billion people [2,3]. Reliable and regular subnational information on the area under production is an essential part of many countries' national accounting process, but statistical methods cannot always meet the needs of food security research and policy [4,5]. Nevertheless, this same information is the basis of policy decisions related to imports, exports and prices, which directly impact food security, especially amongst the poor [6-8].

Remote sensing promises scalable, low-cost and unbiased estimates of rice area to support, augment, improve or even replace survey and statistical methods [5]. However, there are technical challenges for the development of national-scale, operational, remote sensing-based rice crop information systems in Asia:

(i) More than $70 \%$ of the production is during the monsoon or rainy season [9], in which cloud cover is extensive and pervasive [10].

(ii) Rice can be grown under a wide range of conditions and environments and, thus, is found in all corners of Asia [1,9].

(iii) Rice cultivation is dominated by smallholders with field (paddy) sizes that are usually less than two hectares and, in many cases, less than one hectare [11].

(iv) These paddies can form large contiguous areas, especially in coastal areas and deltas [4,9], but the tropical and subtropical climate in much of Asia means that rice can be cultivated with diverse cropping calendars and practices over very short distances $[1,12]$.

The issue of cloud cover (i) can be addressed by using Synthetic Aperture Radar (SAR) imagery, and there is substantial literature on the suitability of SAR for rice crop mapping in the region (see Section 1.2). Optical images can complement SAR, but they cannot be relied upon as the main information source. Even with multi-temporal compositing, many consecutive days or weeks can be lost, which is 
particularly problematic for algorithms that rely on the detection of agronomic flooding at the start of the growing season [4,13]. The wide geographic distribution of rice across Asia (i) necessitates wall-to-wall coverage to adequately capture the 144 million hectares [2] of rice area and suggests that automated or low-level supervised processing is required to do this. Automated processing would also be suited to low-cost cloud computing platforms and low-level operator supervision, which could offset the common bottlenecks of infrastructure, bandwidth and human capacity in emerging economies. The wide range of practices and environments (ii) means that rice detection algorithms should be generalizable and robust [13]. Such methods should show high skill for irrigated and rainfed rice, for short $(<110$ day), medium (110-130 day) and long (>130 day) maturities [1], and for different establishment practices, such as direct seeding or transplanting. The spatial complexity (iii) of rice environments requires high-resolution imagery, and the temporal complexity (iv) requires high-frequency acquisitions across many months of the year.

In short, the remote-sensing requirements are non-trivial, and they go some way towards explaining the dearth of operational rice crop monitoring systems. However, recent and planned launches of SAR sensors coupled with state-of-the-art automated processing can provide sustainable solutions to this challenge to map and monitor one of the world's most important crops.

This aim of this paper is to develop and test a rice area mapping method across multiple sites based on the knowledge of the temporal development of the rice crop under different conditions and its relation to backscatter. Specifically, the novelty in this paper is the development of a rule-based classification approach and parameter selection approach in which the rules and parameters are derived from agronomic knowledge of the rice crop and its management. Thus, the rules and parameters can be easily understood and fine-tuned by users using site-specific knowledge. The purpose here is not to derive the best possible rice map at each site through intensive calibration or large-scale fieldwork, but to introduce a simple approach that is robust, repeatable and suitable for rapid rice mapping over large extents with cost-effective field work.

The overarching goal is to demonstrate that SAR-based operational mapping of rice crops across a diverse range of environments is possible based on the increasing availability of multi-temporal SAR data. The paper is a timely contribution to remote-sensing applications for food security, since it presents a method to derive sufficiently accurate rice area maps under different conditions that are typical of the diversity of rice environments in Asia. Our target audience goes beyond the research community and includes the growing community of stakeholders that have a vested interest in operational remote sensing-based systems for food security applications.

We first summarize past research on SAR applications for rice mapping, justify our choice of algorithm and outline the comprehensive geographic scope of this study.

\subsection{A Summary of SAR Research and Applications for Rice Mapping}

SAR data have a proven ability to detect lowland rice systems (both irrigated and rainfed) through the unique temporal signature of the backscatter coefficient (also termed sigma naught or $\sigma^{\circ}$ ) exhibited by the crop. In the past three decades, a significant number of publications have been dedicated to better understanding this relationship and applying it to rice detection and rice monitoring [14-23]. In summary, these studies have shown that: 
- Lower frequencies (L- and C-band) penetrate deeper into the rice plant than higher frequencies, while only higher frequencies (X-band) interact with grain water content and grain weight sufficiently to show a dual-peak signal in $\sigma^{\circ}$ during the rice season $[15,16,18,19]$.

- The maximum $\sigma^{\circ}$ date at the X-band precedes those of the C- and L-band. Moreover, short wavelengths (X-, Ka-, Ku-band), especially at large incident angles, are sensitive enough to detect even very small rice seedlings just after transplanting $[15,19]$.

- The correlation between $\sigma^{\circ}$ and rice biophysical parameters shows that lower frequencies are more closely related to total fresh weight, leaf area index (LAI) and plant height than other parameters $[15,19]$.

- Although $\sigma^{\circ}$ from X-band is poorly correlated with LAI, it is best correlated with panicle biomass. This means that the $\mathrm{X}$-band can be used for a direct assessment of rice grain yield $[22,23]$.

- When using C-band HV polarization, $\sigma^{\circ}$ is most strongly correlated with the fraction of absorbed photosynthetically-active radiation (fAPAR), which is strongly determined by the amount and structure of leaf elements in a canopy. This means that C-band $\sigma^{\circ}$ can provide information equivalent to the normalized difference vegetation index (NDVI) [23].

- The $\sigma^{\circ}$ from VV polarization increases only during the vegetative stage; it is quite stable at the reproductive stage, and it decreases at the ripening stage because of canopy attenuation $[14,18]$.

- The $\sigma^{\circ}$ from HH polarization increases at the reproductive stage and is quite stable at the ripening stage. The temporal trend of $\sigma^{\circ}$ from $\mathrm{HV}$ is similar to $\mathrm{HH}$ [18].

- The HH/VV polarization ratio at the C-and L-band decreases significantly throughout the season and is thus a good descriptor of rice plant age [18].

- The frequency ratios for HH and VV (C-VV/L-VV and C-HH/L-HH) are significantly lower in the latter part of the rice season when thick vegetation canopy hampers wave penetration [18].

- For X-band, the HH/VV polarization ratio continuously changes as a function of phenology during the vegetative and reproductive stages [20].

- For X-band, the HH-VV phase difference is sensitive to early rice plant emergence. Moreover, the use of four polarimetric features derived from coherence coplanar dual-polarization X-band enables the estimation of five phenological stages from a single date scene $[15,20,21]$.

It is clear from the literature that well-understood relationships exist between rice crop characteristics and backscatter coefficients from different wavelengths, and these relationships have been used to derive different types of algorithms for estimating rice crop characteristics from SAR data. In particular, rice signature interpretation has been proposed based on theoretical models [14,18], and supervised classification algorithms [16] or image ratio methods have been used for rice mapping purposes [14,24]. Another approach for sparse time series is to extract temporal features from the data, such as the minimum, maximum or range of values on a pixel-by-pixel basis, relate those to the known temporal dynamics of the rice crop and use that knowledge to classify areas as rice or non-rice [25]. All of these approaches have been demonstrated successfully in the literature. Theoretical models, based on radiative transfer equations, are particularly useful for understanding the complex backscattering mechanisms; however, they require a significant amount of input parameters that are tuned to the surrounding conditions at the test site. Supervised classifiers rely on a substantial set of good-quality training data to ensure a good 
classification, and there is a risk of over-fitting the classification. Image ratio approaches that divide the intensity values from pairs of images are an efficient way to extract information on rice crop dynamics and are particularly useful when the SAR time series has few images or sparse images through the rice season. Temporal feature extraction from sparse SAR time series meets one of our aims, but the method needs to be adapted to use more comprehensive multi-temporal information for rice area mapping.

A further consideration in the choice of algorithm in this study is that very few studies have tested the robustness of rice detection algorithms across a range of conditions and a range of countries. Varietal choice, crop establishment methods and crop management practices can have a significant effect on the structure of both plant and canopy, the duration and growth rate of the plant and water content. This contextual information on the characteristics of different rice cropping systems must be considered in the development of a robust rice detection algorithm for application over diverse rice environments.

For this reason, we propose a rule-based classification approach to rice area mapping that is based on a small number of rules and parameters that can be quickly fine-tuned from site to site and season to season. These rules and parameters are derived from the above-noted relationships between backscatter and the development of the rice crop, and they build on the temporal feature approach [25] by exploiting regularly acquired, multi-temporal SAR information. Conceptually, the classification approach is based on rules that are agronomically meaningful and, thus, easily understood and easily fine-tuned based on the local knowledge of the rice-growing environment and the key rice-growing stages.

\subsection{Rice Growing Stages and Key Characteristics for SAR Based Detection}

Rice in tropical and subtropical Asia is mainly cultivated in irrigated or lowland rainfed conditions. Rice varieties range in duration from 90 to more than 150 days and with three main crop stages: vegetative (from germination to panicle initiation, from 45 to 100 days), reproductive (from panicle initiation to flowering, around 35 days) and maturity (from flowering to mature grain, around 30 days) (Figure 1). The following aspects contribute to the change in space occupied by the rice plants within a three-dimensional space: (1) appearances and growth of leaves from the main stem (culm) and tillers; (2) stem development and elongation; (3) tillering, defined as the production of stems from rice plants; (4) leaf senescence; and (5) panicle and grain development.

Rice leaves appear approximately every four days during the vegetative phase and every seven days during the reproductive phase [26]. Tillering begins at around the three- to four-leaf stage or approximately 10 days after emergence. Leaf production progresses from the bottom to the top part of the canopy, with the later-appearing leaf blades tending to be greater in length. Vertical growth of rice plants in the early growth stages (early tillering phase) is mainly contributed to by the development of new nodes along the main stem. At the later stage of the tillering phase, towards the panicle initiation stage, stem elongation begins and contributes to a rapid increase in the vertical expansion of the rice canopy. Leaf senescence begins at around heading and contributes to the declining leaf area index. In most cases, the rice leaf area index peaks at around heading. At the heading stage, panicle extension occurs when panicle tips emerge from the base of the last leaf, which is known as the flag leaf. Subsequently, panicles will grow in length and volume and will bear developing grains. By the maturity stage, individual panicles for typical modern, short- to medium-duration rice varieties occupy about $20 \%$ of the space in the top layer of the canopy. However, when arranged along with panicles from all rice 
tillers, panicles and grains together occupy about $30 \%$ of the top layer of the canopy. For typical plant heights of around $100 \mathrm{~cm}$, panicle and grain occupy about $20 \mathrm{~cm}$ of the top layer in the canopy. During the ripening stage of grain development, lasting 25-35 days, the overall rice plant's water content decreases [14].

Figure 1. Rice crop stages. Image from the International Rice Research Institute (IRRI)-Rice Knowledge Bank.

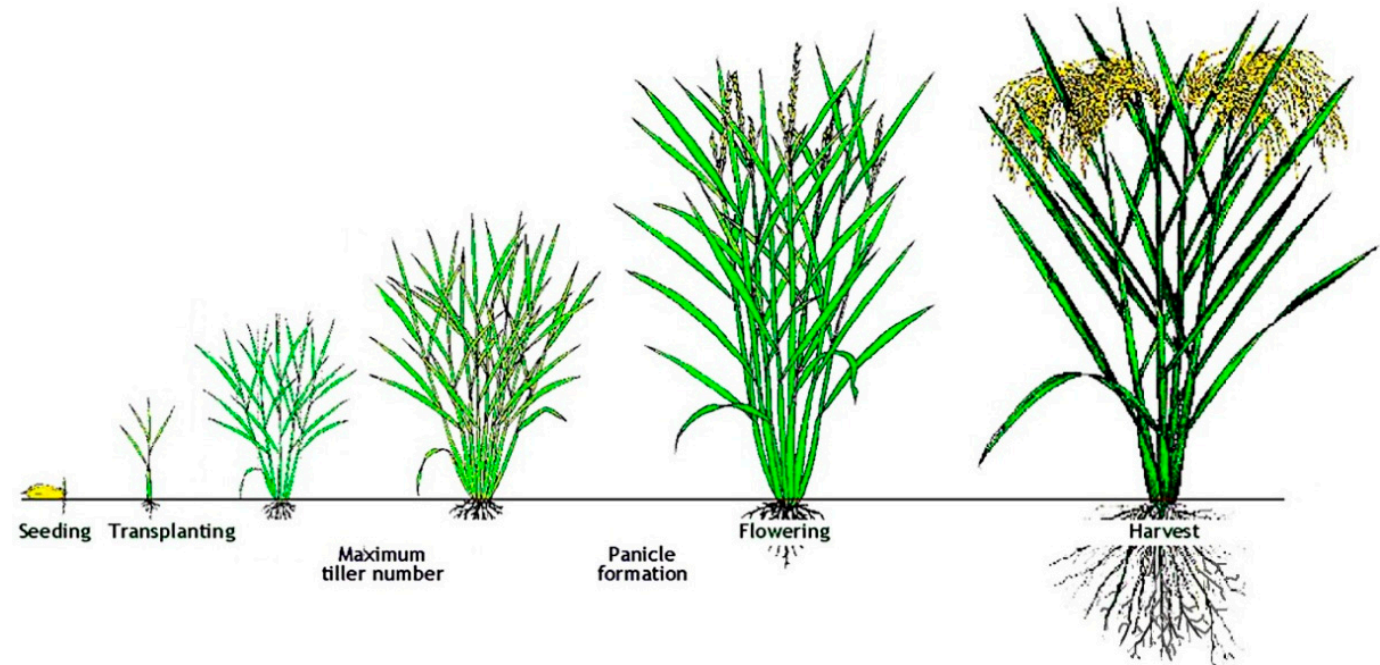

Apart from biology, agronomic management influences biophysical changes in rice plants within the three-dimensional space they occupy, mainly because of the different spacing between rice plants. In the transplanted system, rice plants are grown in a seedbed for about 20 days, and then, the plants are transplanted in a hill configuration at about two plants per hill; a common spacing is $20 \times 20 \mathrm{~cm}$, but this can vary. Prior to transplanting, the rice field is flooded with water at depths ranging from 2 to $15 \mathrm{~cm}$ [14]. This deliberate agronomic flooding is a key element of most remote-sensing rice detection algorithms [13]. After transplanting, the recommended practice is to keep the water level at about $3 \mathrm{~cm}$ and gradually increase it to 5-10 cm with increasing plant height. With rice established using direct seeding, rice seeds are sown at higher density per unit area directly in wet soil or soil with a water level of 2-5 cm [14]. Because of higher population density under direct seeding, the production of tillers is suppressed [26], and thus, the increase in leaf volume within the canopy is mostly contributed to by leaf appearance and growth from the main culms. The water level with the direct-seeding system is kept low until the plants are large enough to withstand shallow flooding at the three- to four-leaf stage. Keeping the water level low during the first 10 days after transplanting or 21 days of direct seeding is recommended for golden snail management, whereas, until about the same time, keeping ample water level in the field is important to suppress weeds.

\subsection{An International Comparison of X-Band SAR Data for Rice Mapping}

The RIICE project-Remote sensing-based Information and Insurance for Crops in Emerging economies - tested SAR-based mapping of rice area across six countries (India, Thailand, Cambodia, Vietnam, Indonesia and the Philippines) between late 2012 and early 2014 (Figure 2). These countries account for $51.5 \%$ of the world's rice area and $45.9 \%$ of production, and they include both rice net 
exporters (Thailand, Vietnam, India and Cambodia) and rice net importers (Indonesia and the Philippines) [2]. These countries are representative of the wide range of geographies, climates and crop practices in subtropical and tropical Asian rice systems.

Figure 2. Location of the 13 SAR footprints in Asia in the RIICE (Remote Sensing-based Information and Insurance for Crops in Emerging economies) project. Country names are shown only where there are footprints. Numbers refer to the site ID used in Table 1.

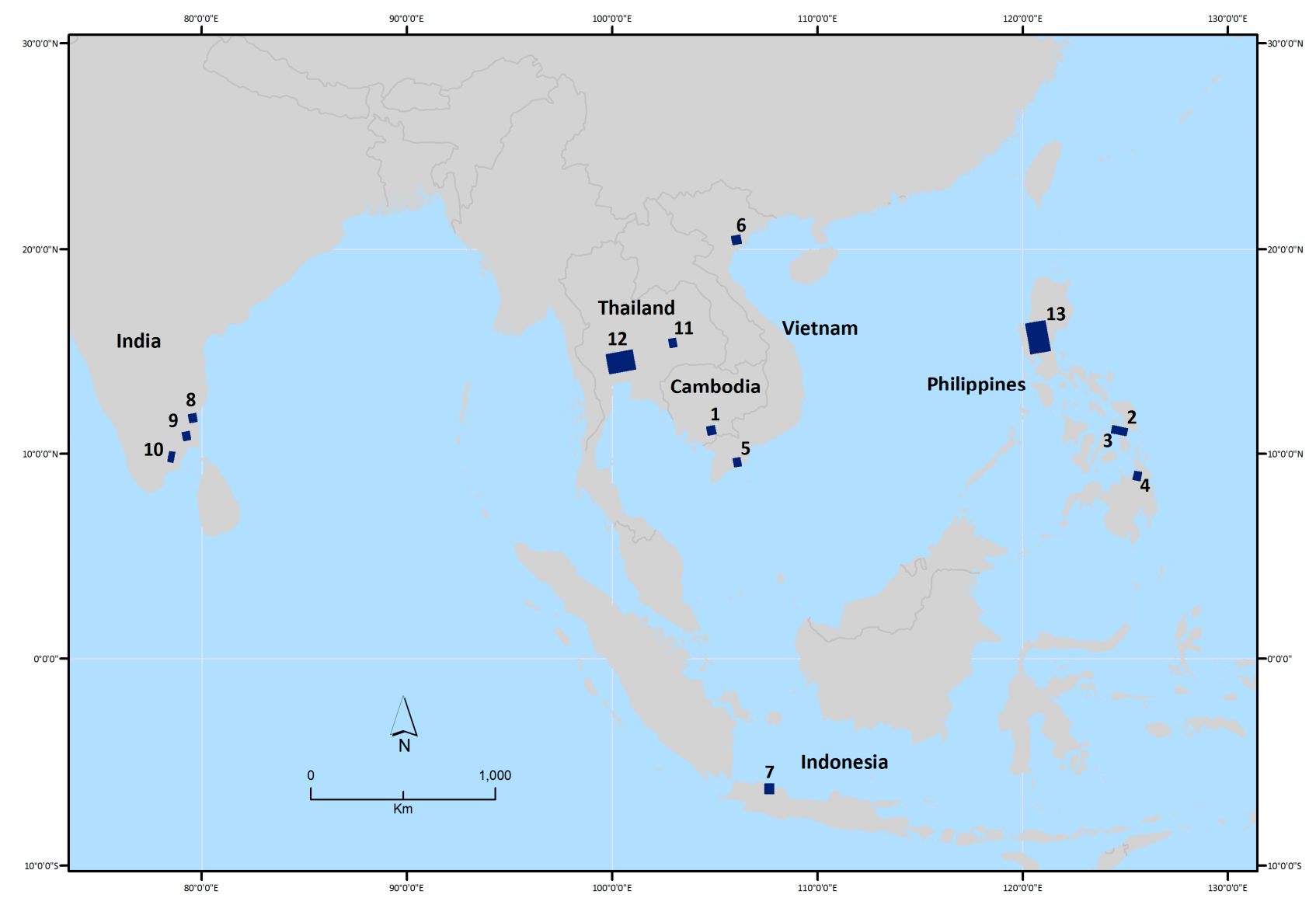

This international collaborative effort used multi-temporal X-band SAR data, semi-automated processing chains, in-season field monitoring and end-of-season validation points to map rice crops across technical demonstrations in six countries (Figure 1). This study uses a greater number of images (127) and number of sites (13) and covers a larger area (4.78 $\mathrm{m} \mathrm{ha}$ ) than any previous assessment of SAR-based remote sensing for rice mapping (Table 1). It is a timely contribution to the literature on the use of SAR for large-scale mapping of rice in Asia for food security applications.

The following sections describe the sites, data, methods and results. The discussion section includes our observations on the strengths and weaknesses of this approach and the challenges faced in conducting the research. We also suggest some criteria and prerequisites for developing SAR acquisition plans that would ensure that remote sensing supports policies for food security, import/export decisions and price stabilization for the world's most important crop. 
Table 1. SAR data acquisition summary: locations, dates and modes. Further details on the acquisitions for each site are included in the Supplemental Information section. CSK, COSMO-SkyMed; TSX, TerraSAR-X.

\begin{tabular}{|c|c|c|c|c|c|c|c|}
\hline Site ID & Country and Study Site & Start and End Dates & $\begin{array}{c}\text { \# of } \\
\text { Images }\end{array}$ & Satellite * & $\begin{array}{c}\text { Scene Center, } \\
\text { Area }\left(\mathbf{k m}^{\mathbf{2}}\right) \\
\end{array}$ & $\begin{array}{c}\text { Mode, Resolution } \\
\text { (m) } \\
\end{array}$ & $\begin{array}{c}\text { Polarization, Angle } \\
\left(\left(^{\circ}\right)\right.\end{array}$ \\
\hline 1 & Cambodia, Takeo & $\begin{array}{c}15 \text { October } 2012 \\
15 \text { April } 2013 \\
\end{array}$ & 12 & CSK & $\begin{array}{c}11.16^{\circ} \mathrm{N}-104.83^{\circ} \mathrm{E} \\
1600\end{array}$ & Stripmap, 3 & $\mathrm{HH}, 40$ \\
\hline 2 & Philippines, Leyte East & $\begin{array}{c}15 \text { May } 2013 \\
20 \text { September } 2013\end{array}$ & 10 & CSK & $\begin{array}{c}11.11^{\circ} \mathrm{N}-124.89^{\circ} \mathrm{E} \\
1600\end{array}$ & Stripmap, 3 & $\mathrm{HH}, 46$ \\
\hline 3 & Philippines, Leyte West & $\begin{array}{c}12 \text { May } 2013 \\
24 \text { September } 2013\end{array}$ & 9 & CSK & $\begin{array}{c}11.18^{\circ} \mathrm{N}-124.56^{\circ} \mathrm{E} \\
1600\end{array}$ & Stripmap, 3 & $\mathrm{HH}, 48$ \\
\hline 4 & $\begin{array}{c}\text { Philippines, } \\
\text { Agusan del Norte }\end{array}$ & $\begin{array}{c}27 \text { May } 2013 \\
2 \text { October } 2013\end{array}$ & 9 & CSK & $\begin{array}{c}8.93^{\circ} \mathrm{N}-125.59^{\circ} \mathrm{E} \\
1600\end{array}$ & Stripmap, 3 & HH, 39 \\
\hline 5 & $\begin{array}{l}\text { Vietnam, } \\
\text { Soc Trang }\end{array}$ & $\begin{array}{c}5 \text { June } 2013 \\
25 \text { September } 2013\end{array}$ & 8 & CSK & $\begin{array}{c}9.60^{\circ} \mathrm{N}-106.09^{\circ} \mathrm{E} \\
1600\end{array}$ & Stripmap, 3 & $\mathrm{HH}, 46$ \\
\hline 6 & $\begin{array}{l}\text { Vietnam, } \\
\text { Nam Dinh }\end{array}$ & $\begin{array}{c}26 \text { May } 2013 \\
17 \text { October } 2013\end{array}$ & 11 & CSK & $\begin{array}{c}20.47^{\circ} \mathrm{N}-106.05^{\circ} \mathrm{E}, \\
1600\end{array}$ & Stripmap, 3 & $\mathrm{HH}, 40$ \\
\hline 7 & Indonesia, Subang & $\begin{array}{c}26 \text { November } 2013 \\
19 \text { April } 2014 \\
\end{array}$ & 9 & CSK & $\begin{array}{c}6.55^{\circ} \mathrm{S}-107.66^{\circ} \mathrm{E}, \\
1600 \\
\end{array}$ & Stripmap, 3 & $\mathrm{HH}, 46$ \\
\hline 8 & $\begin{array}{c}\text { India, Tamil Nadu, } \\
\text { Cuddalore }\end{array}$ & $\begin{array}{l}16 \text { August } 2013 \\
7 \text { January } 2014\end{array}$ & 10 & CSK & $\begin{array}{c}11.74^{\circ} \mathrm{N}-79.56^{\circ} \mathrm{E} \\
1600\end{array}$ & Stripmap, 3 & $\mathrm{HH}, 44$ \\
\hline 9 & $\begin{array}{c}\text { India, Tamil Nadu, } \\
\text { Thanjavur }\end{array}$ & $\begin{array}{c}16 \text { August } 13 \\
26 \text { December } 13\end{array}$ & 9 & CSK & $\begin{array}{c}10.87^{\circ} \mathrm{N}-79.25^{\circ} \mathrm{E} \\
1600\end{array}$ & Stripmap, 3 & $\mathrm{HH}, 41$ \\
\hline 10 & $\begin{array}{c}\text { India, Tamil Nadu, } \\
\text { Sivaganga }\end{array}$ & $\begin{array}{l}18 \text { August } 2013 \\
19 \text { January } 2014 \\
\end{array}$ & 11 & TSX & $\begin{array}{c}9.86^{\circ} \mathrm{N}-78.50^{\circ} \mathrm{E}, \\
1500\end{array}$ & Stripmap, 3 & $\mathrm{HH}, 44$ \\
\hline 11 & Thailand, Muang Yang & $\begin{array}{c}27 \text { May } 2013 \\
19 \text { November } 2013\end{array}$ & 10 & CSK & $\begin{array}{c}15.44^{\circ} \mathrm{N}-102.95^{\circ} \mathrm{E} \\
1600\end{array}$ & Stripmap, 3 & $\mathrm{HH}, 43$ \\
\hline 12 & Thailand, Suphan Buri & $\begin{array}{c}18 \text { June } 2013 \\
24 \text { October } 2013\end{array}$ & 9 & CSK & $\begin{array}{c}14.53^{\circ} \mathrm{N}-100.44^{\circ} \mathrm{E} \\
14,000\end{array}$ & ScanSAR, 15 & $\mathrm{HH}, 45$ \\
\hline \multirow[t]{2}{*}{13} & Philippines, Nueva Ecija & $\begin{array}{c}25 \text { May } 2013 \\
23 \text { September } 2013\end{array}$ & 10 & TSX & $\begin{array}{c}15.71^{\circ} \mathrm{N}-120.75^{\circ} \mathrm{E} \\
15,000\end{array}$ & ScanSAR, 10 & $\mathrm{HH}, 45$ \\
\hline & $\begin{array}{c}\text { Total number of images } \\
\text { and footprint area }\end{array}$ & & 127 & & 46,500 & & \\
\hline
\end{tabular}

* All acquisitions are in the X-band.

\section{SAR Data, Field Data and Study Sites}

\subsection{SAR Data}

Multi-temporal X-band SAR Single Look Complex (SLC) data were obtained from the Italian Space Agency (ASI/e-GEOS) and GISTDA (Geo-informatics and Space Technology Development Agency) for COSMO-SkyMed (CSK) data and from InfoTerra GmbH for TerraSAR-X (TSX) data. In all cases, data were obtained in $\mathrm{HH}$ polarization with consistent incidence angles in each multi-temporal stack, ranging from 39 to 48 degrees across sites. A large incidence angle is preferred, because (i) wind effects on water (in particular, during land preparation prior to transplanting) are significantly decreased, (ii) the 
dynamic of the radar backscatter is larger and (iii) the spatial resolution is higher. The image acquisition dates, locations, mode, pixel size, polarization and incidence angles are shown in Table 1. Image mode, extent, pixel size, polarization and incidence angles were constant for each footprint. Full details of the acquisitions for each of the 13 sites are included in the Supplemental Information section (Table S1).

CSK data are available from four X-band HH-SAR satellites with a 3.12-cm wavelength and a 16-day revisit period for the same satellite with the same observation angle. We used Stripmap mode (3-m resolution) at 10 sites with a footprint of $40 \times 40 \mathrm{~km}$ and ScanSAR Wideregion mode (15-m resolution) at one site with a footprint of $100 \times 140 \mathrm{~km}$. Acquisition plans were made using one primary satellite from the constellation for each site with backup plans in place for the second, third and fourth satellites in the constellation in the event of a cancellation. These backup acquisitions are one, four and eight days after the primary acquisition pass. Rapid notification of a cancelation is required to change planned field visits to the backup date, but this proved to be challenging to implement consistently through the monitoring campaign whenever backups were required.

TSX is provided by one X-band HH SAR satellite with a 3.11-cm wavelength and 11-day revisit period with the same observation angle. We used Stripmap mode ( $3 \mathrm{~m}$ resolution) at one site with a footprint of $30 \times 50 \mathrm{~km}$ and ScanSAR mode $(10 \mathrm{~m}$ resolution) at one site with a footprint of $100 \times 150 \mathrm{~km}$. No backup plan was implemented for TSX.

\subsection{Field Observations for Calibration of the Rice Detection Algorithm and Map Validation}

Field observations were performed throughout the season in up to 20 paddy fields within each footprint. These fields were selected, with the farmers' consent, prior to the start of the rice season and the image acquisition schedule. Observations were made on or as close to the image acquisition date as possible, depending on national holidays or other events that prevented easy access to locations. Observations included latitude and longitude from handheld GPS receivers, descriptions and photos of the status of the field, plant height, water depth, weather conditions, crop stage and leaf area index (LAI). The same field data collection protocols were used at all sites. LAI measurements were taken only during visits between seedling and flowering stages, and these were recorded non-destructively using the same equipment at each site: an AccuPAR LP-80 Ceptometer (Decagon Devices, Inc., Pullman, WA, USA). The specific model of the GPS and digital cameras varied across sites. At the end of the season, the farmer was interviewed to collect information on the rice variety, water source, crop management and establishment practices, as well as inputs, such as pesticide and fertilizer.

In total, 228 locations were regularly monitored across the 13 footprints, with 1922 separate visits made to these locations to collect in-season information on the status of the rice crop, an average of 8 visits per location and 18 locations per footprint (Table 2).

A validation exercise was conducted for each footprint to assess the accuracy of the rice classification. We first considered the regular sampling grid and random selection of locations as appropriate methods to select representative and spatially well-distributed samples. However, these approaches were quickly deemed too time consuming and too expensive to apply over 13 sites covering 46,500 $\mathrm{km}^{2}$. For these reasons, a rapid land cover appraisal method was adopted to collect land cover information at approximately 100 locations throughout each footprint with these points split 50/50 between non-rice points and rice points. This conforms to the minimum number of samples per land cover class accounting 
for both statistical sampling requirements [27] and practical considerations, given the available resources and accessibility.

Table 2. Summary of site visits and observed rice crop characteristics during the monitored seasons.

\begin{tabular}{|c|c|c|c|c|c|c|c|c|}
\hline $\begin{array}{l}\text { Site } \\
\text { ID }\end{array}$ & $\begin{array}{l}\text { Country, } \\
\text { Study Site }\end{array}$ & Season & $\begin{array}{l}\text { Period } \\
\text { Covered }\end{array}$ & $\begin{array}{c}\text { Number of } \\
\text { Fields, Visits }\end{array}$ & $\begin{array}{c}\text { Crop } \\
\text { Establishment } \\
\text { Method } \\
\end{array}$ & $\begin{array}{l}\text { Variety and Maturity } \\
\text { (days) }\end{array}$ & $\begin{array}{c}\text { Water } \\
\text { Management }\end{array}$ & Notes \\
\hline 1 & $\begin{array}{l}\text { Cambodia, } \\
\text { Takeo }\end{array}$ & Dry & $\begin{array}{l}\text { October to } \\
\text { April }\end{array}$ & $\begin{array}{l}4 \text { fields, } \\
20 \text { visits }\end{array}$ & Direct seeding & IR504 (95) & Irrigated & \\
\hline 2 & $\begin{array}{l}\text { Philippines, } \\
\text { Leyte East }\end{array}$ & Wet & $\begin{array}{c}\text { May to } \\
\text { September }\end{array}$ & $\begin{array}{l}20 \text { fields, } \\
200 \text { visits }\end{array}$ & Transplanting & NSIC Rc222 (114) & Irrigated & $\begin{array}{c}\text { Typhoon on } 8 \\
\text { November } 2013\end{array}$ \\
\hline 3 & $\begin{array}{l}\text { Philippines, } \\
\text { Leyte West }\end{array}$ & Wet & $\begin{array}{c}\text { May to } \\
\text { September }\end{array}$ & $\begin{array}{l}20 \text { fields, } \\
200 \text { visits }\end{array}$ & Transplanting & $\begin{array}{l}\text { NSIC Rc216 (112), } \\
\text { NSIC Rc238 (110) }\end{array}$ & Irrigated & \\
\hline 4 & $\begin{array}{l}\text { Philippines, } \\
\text { Agusan del } \\
\text { Norte }\end{array}$ & Dry & $\begin{array}{l}\text { May to } \\
\text { October }\end{array}$ & $\begin{array}{l}18 \text { fields, } \\
182 \text { visits }\end{array}$ & $\begin{array}{l}\text { Transplanting } \\
\text { and direct } \\
\text { seeding } \\
\end{array}$ & $\begin{array}{l}\text { PSB Rc18 (123), } \\
\text { NSIC Rc160 (107), } \\
\text { NSIC Rc122 (112) }\end{array}$ & $\begin{array}{l}\text { Irrigated with } \\
\text { some rainfed }\end{array}$ & \\
\hline 5 & $\begin{array}{l}\text { Vietnam, } \\
\text { Soc Trang }\end{array}$ & $\begin{array}{l}\text { Summer- } \\
\text { autumn }\end{array}$ & $\begin{array}{c}\text { June to } \\
\text { September }\end{array}$ & $\begin{array}{l}12 \text { fields, } \\
66 \text { visits }\end{array}$ & $\begin{array}{l}\text { Transplanting } \\
\text { and direct } \\
\text { seeding }\end{array}$ & $\begin{array}{c}\text { OM6976 (100), } \\
\text { OM3673 }(95), \\
\text { ST5 }(120), \\
\text { OM108-5 (100), } \\
\text { OM9584-1 (95), } \\
\text { OM4900 (100) }\end{array}$ & Irrigated & \\
\hline 6 & $\begin{array}{l}\text { Vietnam, } \\
\text { Nam Dinh }\end{array}$ & Summer & $\begin{array}{c}\text { July to } \\
\text { November }\end{array}$ & $\begin{array}{l}20 \text { fields, } \\
160 \text { visits }\end{array}$ & Transplanting & $\begin{array}{c}\text { Tap Giao (125), } \\
\text { BC } 15(134)\end{array}$ & Irrigated & \\
\hline 7 & $\begin{array}{l}\text { Indonesia, } \\
\text { Subang }\end{array}$ & Wet & $\begin{array}{l}\text { November } \\
\text { to April }\end{array}$ & $\begin{array}{l}20 \text { fields, } \\
160 \text { visits }\end{array}$ & Transplanting & $\begin{array}{c}\text { Ciherang, Inpari, } \\
\text { Mekonga, } \\
\text { Sintanur (115), } \\
\text { Ketan, IR42 (135) }\end{array}$ & Irrigated & $\begin{array}{l}\text { Early drought } \\
\text { with flood } \\
\text { event, early } \\
\text { January } 2014\end{array}$ \\
\hline 8 & $\begin{array}{c}\text { India, } \\
\text { Tamil Nadu, } \\
\text { Cuddalore }\end{array}$ & Samba & $\begin{array}{l}\text { mid-July to } \\
\text { January }\end{array}$ & $\begin{array}{l}20 \text { fields, } \\
160 \text { visits }\end{array}$ & Transplanting & $\begin{array}{l}\text { CR1009 (160), } \\
\text { BPT5204 (135), } \\
\text { White Ponni (130), } \\
\text { Co } 50(160)\end{array}$ & Irrigated & \\
\hline 9 & $\begin{array}{c}\text { India, } \\
\text { Tamil Nadu, } \\
\text { Thanjavur }\end{array}$ & Samba & $\begin{array}{l}\text { August to } \\
\text { December }\end{array}$ & $\begin{array}{l}20 \text { fields, } \\
162 \text { visits }\end{array}$ & $\begin{array}{l}\text { Transplanting } \\
\text { and direct } \\
\text { seeding }\end{array}$ & $\begin{array}{l}\text { CR1009 (160), } \\
\text { BPT5204 (135), } \\
\text { ADT } 50(160)\end{array}$ & Irrigated & \\
\hline 10 & $\begin{array}{c}\text { India, } \\
\text { Tamil Nadu, } \\
\text { Sivaganga }\end{array}$ & Samba & $\begin{array}{l}\text { September } \\
\text { to January }\end{array}$ & $\begin{array}{l}18 \text { fields, } \\
110 \text { visits }\end{array}$ & $\begin{array}{l}\text { Transplanting } \\
\text { and direct } \\
\text { seeding }\end{array}$ & $\begin{array}{l}\text { ADT45 (110), } \\
\text { JGL (100-110), } \\
\text { ADT36 (110), } \\
\text { Jothi (110) }\end{array}$ & Semi-dry rice & $\begin{array}{c}\text { Moisture stress } \\
\text { and maturity }\end{array}$ \\
\hline 11 & $\begin{array}{c}\text { Thailand, } \\
\text { Muang Yang }\end{array}$ & Wet & $\begin{array}{l}\text { May to } \\
\text { November }\end{array}$ & $\begin{array}{l}16 \text { fields, } \\
130 \text { visits }\end{array}$ & Direct seeding & $\begin{array}{l}\text { KDML105 (>150), } \\
\text { RD15 (179), } \\
\text { RD6 (178) }\end{array}$ & Rainfed & $\begin{array}{l}\text { Early drought } \\
\text { with flood } \\
\text { event, early } \\
\text { October } 2013\end{array}$ \\
\hline
\end{tabular}


Table 2. Cont.

\begin{tabular}{|c|c|c|c|c|c|c|c|c|}
\hline $\begin{array}{l}\text { Site } \\
\text { ID }\end{array}$ & $\begin{array}{l}\text { Country, } \\
\text { Study Site }\end{array}$ & Season & $\begin{array}{c}\text { Period } \\
\text { Covered }\end{array}$ & $\begin{array}{l}\text { Number of } \\
\text { Fields, Visits }\end{array}$ & $\begin{array}{c}\text { Crop } \\
\text { Establishment } \\
\text { Method }\end{array}$ & $\begin{array}{l}\text { Variety and Maturity } \\
\text { (days) }\end{array}$ & $\begin{array}{c}\text { Water } \\
\text { Management }\end{array}$ & Notes \\
\hline 12 & $\begin{array}{l}\text { Thailand, } \\
\text { Suphan Buri }\end{array}$ & Wet & $\begin{array}{l}\text { June to } \\
\text { October }\end{array}$ & $\begin{array}{l}20 \text { fields, } \\
172 \text { visits }\end{array}$ & Direct seeding & $\begin{array}{l}\text { RD31 (115), } \\
\text { RD47 (112), } \\
\text { RD41 (115), } \\
\text { PTT1 (120), } \\
\text { RD29 (95), } \\
\text { SPR1 (115), } \\
\text { PLK2 (92) }\end{array}$ & Irrigated & \\
\hline 13 & $\begin{array}{l}\text { Philippines, } \\
\text { Nueva Ecija } \\
\end{array}$ & Wet & $\begin{array}{c}\text { July to } \\
\text { November }\end{array}$ & $\begin{array}{l}20 \text { fields, } \\
200 \text { visits }\end{array}$ & Transplanting & NSIC Rc222 (114) & Irrigated & \\
\hline & $\begin{array}{c}\text { Total number } \\
\text { of fields and } \\
\text { visits } \\
\end{array}$ & & & $\begin{array}{l}228 \text { fields, } \\
1922 \text { visits }\end{array}$ & & & & \\
\hline
\end{tabular}

Field staff conducted multi-day journeys through each footprint, following routes based on local knowledge of the land cover. GPS coordinates, photos and land cover descriptions were collected at each location. These map validation assessments were generally conducted in-season, in the reproductive or ripening stage before harvesting, but in some cases, the assessment was conducted post-season; hence, rice stubble and farmer surveys were used to confirm that the observed post-harvest situation reflected the presence of a rice crop during the monitored season. Locations were chosen such that the land cover was homogeneous in a $15-\mathrm{m}$ radius around each GPS point for sites using 3-m resolution imagery and a 50-m radius for sites using $10-\mathrm{m}$ or $15-\mathrm{m}$ resolution imagery.

\subsection{Study Site Characteristics}

The 13 monitoring sites cover a range of environments, crop establishment methods, water management practices and varietal maturities that are typical of rice-growing areas in South and Southeast Asia. We do not consider the harvesting method nor residual stubble, since most of the acquisitions and field observations stopped before harvest. The footprints cover irrigated and rainfed lowland systems that account for more than $90 \%$ of the rice-growing area [9]. Deepwater and upland rice account for the remaining small proportion of the area and are not represented in this study. Monitoring at 10 sites was done in the wet or monsoon season (Sites 2, 3 and 6-13), whereas three sites were monitored in the dry season (Sites 1, 4 and 5). Six sites established the rice crop by transplanting (Sites 2, 3, 6-8 and 13); four sites had a mixture of transplanting and direct seeding (Sites 4, 5, 9 and 10); and three established the crop by direct seeding (Sites 1, 11 and 12). Ten sites were in irrigated areas (Sites 1-3, 5-9, 12 and 13); one site was mainly irrigated with some rainfed area (Site 4); one site practiced semi-dry rice, which is rainfed at the start of the season and irrigated in later crop stages (Site 10); and one site was purely rainfed (Site 11). Varietal maturities ranged from short at one site (Site 1) to a mixture of short to medium at two sites (Sites 5 and 10), medium at seven sites (Sites 2-4, 6, 7 and 12), long at two sites (Sites 8 and 9 ) and very long at one site (Site 11). Table 2 summarizes the characteristics of the dominant rice systems 
covered by each footprint, and further details for each site and monitored season are expanded upon below.

Cambodia (Site 1, Takeo): In this area, farmers typically grow short-duration non-photoperiod-sensitive varieties (such as IR504) for the dry season, early wet season and recession rice. Improved varieties, such as Phka Romduol and local varieties with medium and long growth durations, are grown in the main wet season. Monitoring was conducted in four locations (three from Angkor Borey and one in Prey Kabas District) during the recession rice of October 2012, to April 2013. Farmers started broadcasting their fields as soon as the water receded from October to November. The variety used by farmers in this area was mainly IR504. Groundwater was used as supplementary irrigation when there was a lack of access to water from river or other irrigation sources.

Philippines (Sites 2, 3, 4 and 13, Leyte East, Leyte West, Agusan del Norte and Nueva Ecija): These sites represent three geographic areas of the country-Luzon, Visayas and Mindanao, respectively_characterized by different rice cropping practices. The four municipalities monitored in Nueva Ecija were San Jose, Sto. Domingo, Talavera and Aliaga. They were classified as irrigated lowland, and they rely mainly on the release of irrigation water from the Upper Pampanga River Integrated Irrigation System (UPRIIS). Rice plots of the monitoring locations usually maintain flooded soil moisture with a 3-6-cm water level from the vegetative to the reproductive stage. Most of the 20 monitoring locations have a rice-rice cropping pattern, which was described as planting rice for two cropping seasons, wet and dry. Crop establishment during the wet season, 2013, in Nueva Ecija started in July. Rice farmers in the monitoring locations mostly planted NSIC Rc 222, an inbred variety with a duration of 114 days. This variety was popular because of its high-yielding capacity, resistance to pests and resistance to lodging.

In Leyte, the municipalities were Matag-ob, Kananga and Ormoc City in the west and Dulag, Tolosa, Tanauan, Palo, Sta. Fe and Alang-alang in the east. The rice-rice cropping system is widely practiced in the province. Crop establishment in both east and west started in May, and the crop was harvested in September/October (wet season), with most of the farmers practicing transplanting. Most of the locations were irrigated, with the irrigation supply largely depending on the Bao, Binahaan, Pongso, Gibuga, Suong-Tibak and Mainit river irrigation systems. Varieties planted included NSIC Rc 222, NSIC Rc 238 and NSIC Rc 216, with a duration ranging from 110 to 114 days. Leyte was severely affected by "super typhoon" Haiyan (named Yolanda in the Philippines) on 8 November 2013, which passed directly over the region. Areas in eastern Leyte had already harvested by this date, while harvesting was in progress in western Leyte. Satellite observations, monitoring data and field validation data relevant to this study were all completed prior to this devastating typhoon.

In Agusan del Norte, the monitoring locations for the dry season, 2013 (May to October), were found in the municipalities of Bayugan, Buenavista, Butuan City and Cabadbaran City. These areas are classified as irrigated lowland and mainly rely on the schedule of the release of irrigation water from the Cabadbaran and Taguibo river irrigation systems and the Aupagan area. However, some locations are also rainfed. Farmers with a regular irrigation supply maintain a 3-7-cm water level in the field during the vegetative and reproductive stages of the rice crop. The most common method of crop establishment is transplanting, but some farmers practice direct seeding. A majority of the farmers in the monitoring locations prefer PSB Rc18, NSIC Rc122 and NSIC Rc 160 rice varieties. 
Vietnam (Sites 5 and 6, Soc Trang and Nam Dinh): The lower Mekong is one of the most well-studied rice areas in the world, with multiple examples of SAR applications. In the 2013 summer-autumn (June to September) rice monitoring in Soc Trang Province, a majority of the farmers practiced direct seeding. OM rice varieties (Cuu Long Delta Rice Research Institute, Omon District, Cantho Province, Vietnam), such as OM4900, OM6976, OM1085, etc., were the dominant rice varieties used, with durations ranging from 95 to 110 days. Most of the rice fields that were monitored were irrigated areas.

In the Red River Delta, the selected locations mostly covered Ha Nam and Nam Dinh provinces. Every year, this area is transplanted with two rice crops: spring-rice (February to June) and summer-rice (July to November). Most of the paddies monitored in the 2013 summer-rice season were irrigated. The dominant rice varieties planted in the monitoring locations were Tap Giao (125 days) and BC15 (135 days), and farmers mostly transplanted their rice plants.

Indonesia (Site 7, Subang): Subang District, West Java, is one of the central rice production provinces in Indonesia. Since irrigation water is available year-round, farmers can grow rice in any month of the year. During the wet season, 2013/2014 (November to April), a total of 20 locations were monitored. In the monitoring locations, the main rice varieties used by farmers during the wet season were Ciherang, Inpari, Mekonga, Sintanur (115 days), IR42 and Ketan (135 days), and the common crop establishment method was transplanting. Flooding was observed in some parts of the district in early January 2014, but this did not have a significant impact on the rice crop.

India (Sites 8, 9 and 10, Cuddalore, Thanjavur and Sivaganga): In Cuddalore District, the monitoring covered the samba season from mid-July 2013, to the first week of January 2014. The majority of the monitored locations were irrigated, predominantly from groundwater (wells). Popular rice varieties grown were CR1009, BPT5204 and White Ponni, with maturity durations ranging from 135 to 160 days. Both transplanting and direct seeding of rice are common in this district, with the former establishment method being more dominant.

Thanjavur is popularly known as the "Rice Bowl" of Tamil Nadu and "Granary of South India", as it is the major district contributing to the food grain supply of the state. The samba season, 2013, from August to December, was monitored. Most of the monitored locations were irrigated, and farmers practiced transplanting and direct seeding as their crop establishment method. Medium- and long-duration varieties, such as CR1009, BPT5204 and ADT (R) 50, were mainly grown, with durations from 135 to 160 days.

In Sivaganga, the samba season lasted from September 2013, to January 2014. Rice cultivation was broadly grouped into three types: transplanted, semi-dry and direct seeded. The transplanted system was practiced in the blocks of Thirupuvanam, Sivaganga, Manamadurai, Singampunari, Thirupattur, S. Pudur, Sakkottai, Kallal and Illayangudi. In the semi-dry rice system, seeds are pre-monsoon sown and are under rainfed conditions for 30-45 days. Later, the fields were converted into wet fields by irrigating from tanks, and this type of cultivation was practiced in the blocks of Sakkottai, Kannankudi, Devakkottai, Kallal, Kalayarkovil, Sivaganga, Manamadurai, Illayangudi and Thirupattur. Direct-seeded rice cultivation mainly depends on rainfall and is mostly practiced in Illayangudi, Devakkotai, Kannankudi and Kalaiyarkovil blocks. Short-duration rice varieties, such as ADT36, ADT45 and JGL, were popularly grown in the monitoring locations. 
Thailand (Sites 11 and 12, Muang Yang and Suphan Buri): In Muang Yang District in Nakorn Ratchasima Province, in the northeast region of Thailand, 16 locations were monitored between May and November 2013. In general, rice farmers rely on rainfall as their main water source for rice fields, resulting in relatively homogeneous crop establishment dates. Farmers typically practice direct seeding using photoperiod-sensitive varieties, such as KDML105, RD 6 and RD 15, whose maturity varies from 150 to 178 days.

Meanwhile, from June to October 2013, 20 locations were monitored in Suphan Buri Province (located in the Chao Phraya Central Plain), where non-photoperiod-sensitive rice varieties were grown in irrigated and intensive rice production systems. Irrigation water is available throughout the year, and the crop establishment date can vary widely over small distances. The rice varieties used by farmers in Suphan Buri included RD 31, RD 47, RD 41, PTT1, RD29, SPR1 and PSK2. These are mostly short-duration varieties with maturity durations ranging from 92 to 120 days.

\section{Methods}

The following steps were implemented and are described below in more detail. First, the SAR time-series data underwent a series of basic processing steps to generate terrain-geocoded $\sigma^{\circ}$ values suitable for analysis. This multi-temporal stack was analyzed using a rule-based classifier to detect rice areas. The rules for the classifier are based on a small number of parameters that must be selected by the operator or user. Temporal feature descriptors are derived from temporal signatures in the monitored fields and used to guide the user in setting these parameters for each site. In this stage, it is clear that there must always be a degree of user expertise in the setting of the parameters, relying on expert local knowledge or other sources of information to further guide the parameter values. Finally, the accuracy of the rice area maps is assessed against field data.

\subsection{Basic Processing of SAR Data for Multi-Temporal Analysis}

A fully automated processing chain was developed to convert the multi-temporal space-borne SAR SLC data into terrain-geocoded $\sigma^{\circ}$ values. The processing chain is a module within the MAPscape-RICE software [25]. The basic processing chain includes the following steps:

1. Strip mosaicking: To facilitate the overall data processing and data handling, single frames of the same orbit and acquisition date were mosaicked along their azimuth, generating long strips in slant range geometry. This step is performed exclusively when the SAR data are zero-Doppler focused.

2. Co-registration: Images acquired with the same observation geometry and mode were co-registered in slant range geometry. The co-registration was performed in three steps: (i) a gross shift estimation based on the orbital data; (ii) a set of subwindows was automatically identified based on a reference image and on the images to be co-registered, and subsequently, the shifts between pixels of corresponding subwindows were calculated, including elevation by means of cross-correlation; (iii) finally, the shifts to be applied in the azimuth direction and range direction were calculated by a polynomial function depending on the pixel position, respectively, in the azimuth and range. 
3. Time-series speckle filtering: Within the multi-temporal filtering, an optimum weighting filter was applied to balance differences in reflectivity between images at different times [28]. Multi-temporal filtering is based on the assumption that the same resolution element on the ground is illuminated by the radar beam in the same way and corresponds to the same slant range coordinates in all images of the time series. The reflectivity can change from one time to the next because of a change in the dielectric and geometrical properties of the elementary scatters, but should not change because of a different position of the resolution element with respect to the radar.

4. Terrain geocoding, radiometric calibration and normalization: A backward solution by considering a digital elevation model (DEM) was used to convert the positions of the $\sigma^{\circ}$ elements into slant range image coordinates. A range-Doppler approach was applied to convert the two-dimensional row and column coordinates of the slant range image into three-dimensional object coordinates in a given cartographic reference system. During this step, the radiometric calibration was performed by means of the radar equation, in which scattering area, antenna gain patterns and range spread loss were considered. Finally, in order to compensate for the range dependency, $\sigma^{\circ}$ was normalized according to the cosine law of the incidence angle.

5. Anisotropic non-linear diffusion (ANLD) filtering: This filter significantly smoothes homogeneous targets, while also enhancing the difference between neighboring areas. The filter uses the diffusion equation, in which the diffusion coefficient, instead of being a constant scalar, is a function of image position and assumes a tensor value [29]. In this way, it is locally adapted to be anisotropic close to linear structures, such as edges or lines.

6. Removal of atmospheric attenuation: Although microwave signals have the ability to penetrate clouds, it is possible that $\sigma^{\circ}$ from shorter wavelengths (X-and C-band) can be locally attenuated by water vapor in the range of several $\mathrm{dB}$, because of severe (tropical) storms. The temporal signature of $\sigma^{\circ}$ can be affected by these events in two ways: (i) the thick layer of water vapor generates a strong decrease in $\sigma^{\circ}$ during the event, followed by a strong increase after the event; (ii) the intense rainfall generates a strong increase in $\sigma^{\circ}$ during the event, followed by a strong decrease after the event. These effects were removed by analyzing the temporal $\sigma^{\circ}$ signature: anomalous peaks or troughs were identified, and the $\sigma^{\circ}$ values were corrected by means of an interpolator. The correct application of this process relies strongly on a priori knowledge of the rice crop calendar and the weather conditions when the image was acquired.

\subsection{Multi-Temporal $\sigma^{\circ}$ Rule-Based Rice Detection}

The multi-temporal stack of terrain-geocoded $\sigma^{\circ}$ images was input to a rule-based rice detection algorithm in MAPscape-RICE. The temporal evolution of $\sigma^{\circ}$ is analyzed from an agronomic perspective, which also requires a priori knowledge of rice maturity, calendar and duration and crop practices from field information and knowledge of the study location. The temporal signature is frequency and polarization dependent and also depends on the crop establishment method and, to some extent, on crop maturity. This implies that general rules can be applied to detect rice, but that the parameters for these rules may need to be adapted according to the agro-ecological zone, crop practices and rice calendar. 
Figure 3 gives an overview of the developed rule-based detection algorithm. Details are expanded upon below.

Figure 3. Rule-based rice detection algorithm for multi-temporal X-band $\sigma^{\circ}$ in MAPscape-RICE.

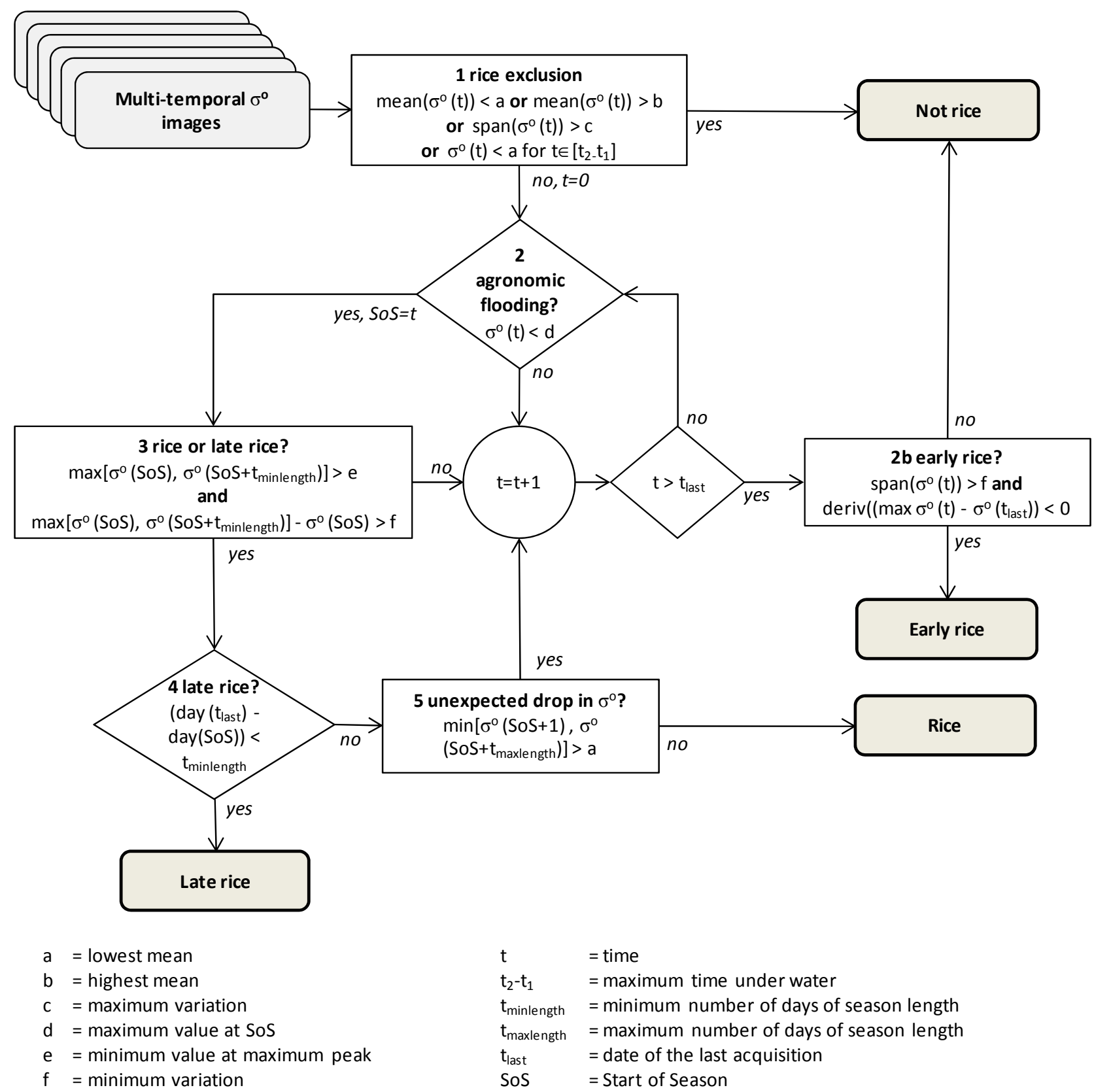

We derive rice area from the SAR temporal signature as follows.

1 The first rule is the rice exclusion condition. The following conditions are applied to the temporal signature for each pixel:

i. Is the average $\sigma^{\circ}$ lower than expected (compared against parameter $a=$ lowest mean)? This masks out areas with consistently low $\sigma^{\circ}$ values that would be typical of stable water bodies. 
ii. Is the average $\sigma^{\circ}$ higher than expected (compared against parameter $b=$ highest mean)? This masks out areas of consistently high $\sigma^{\circ}$ values that would be typical of settlements or infrastructure.

iii. Does $\sigma^{\circ}$ remain under a minimum value longer than expected (compared against parameter $a=$ lowest mean and $t_{2}-t_{1}=$ maximum time under water)? Agronomic flooding of a rice field occurs over a short period of time; any longer duration, but non-permanent flooding, such as fishponds, irrigation tanks or seasonal wetlands, should be removed by this condition.

iv. Is the variation in $\sigma^{\circ}$ larger than expected (compared against parameter $c=$ maximum variation)? Rice, like other seasonal field crops, will show variation in $\sigma^{\circ}$ over time. There is a maximum amount of variation that can be expected from growth in biomass over the season, and this condition removes any areas with unusually high variation.

This exclusion or masking concept is similar to previous paddy mapping with optical time-series data [4], which also stresses the importance of using ancillary data, when available, to mask out non-rice areas a priori. Any pixel that meets one or more of these exclusion conditions is labeled non-rice and excluded from further processing. Any pixel that does not meet any of these conditions has exhibited a temporal signature that is consistent with a rice crop (see Section 1.2) and is retained for further processing.

2 We then apply a stepwise process looking at the temporal signature in more detail starting with a temporal series of data from the first image $(t=0)$ in the time series to the last $\left(t=t_{\text {last }}\right)$. The second rule looks for any evidence of agronomic flooding at the start of the season $(S o S)$. Flooded paddy fields exhibit low $\sigma^{\circ}$ values, so, starting with $t=0$, if $\sigma^{\circ}$ is less than a maximum value $(d=$ maximum value at $S o S)$ at this supposed $S o S$ date, then the pixel is retained for further analysis. If not, we move to the next image in the time series $(t=t+1)$ and apply the same rule again.

$2 \mathrm{~b}$ If the above condition is not met on any $t$ value between 0 and tlast, we apply another rule to determine whether this pixel could be a rice crop that was established before the first date in the time series. In other words, despite not detecting the moment of agronomic flooding, is there enough evidence in the temporal signature to still classify this pixel as rice? This rule is critical in areas where there is considerable heterogeneity in crop establishment dates beyond that which was anticipated in the acquisition plan. The conditions are:

i. Does the variation in $\sigma^{\circ}$ reach a suitable minimum consistent with that expected from a rice crop (compared against parameter $f=$ minimum variation)? This detects any evidence of biomass increase that could be part of a rice crop signature.

ii. Is a negative slope in $\sigma^{\circ}$ detected between the maximum $\sigma^{\circ}$ value in the time series and the $\sigma^{\circ}$ value at $t=t_{\text {last }}$ ? This detects any evidence of a drop in $\sigma^{\circ}$ value in later stages of the season that again would be consistent with knowledge of X-band $\sigma^{\circ}$ temporal signatures for rice.

Any pixel that meets both of these conditions is labeled early rice and excluded from further processing. Any pixel that does not meet both is labeled non-rice and excluded from further processing.

3 Once a flood detection has been made, the next rule looks for further evidence of a rice crop based on: 
i. For the detected $S o S$, does the $\sigma^{\circ}$ signature reach a suitable maximum value consistent with that expected from a rice crop between the detected $S o S$ date and the $t_{\text {minlength }}$ date (compared against parameter $e=$ minimum $\sigma^{\circ}$ value at maximum peak)?

ii. For the detected $S o S$, does the variation in the $\sigma^{\circ}$ signature reach a suitable minimum consistent with that expected from a rice crop between the detected $S o S$ date and the $t_{\text {minlength }}$ date (compared against parameter $f=$ minimum variation)?

Again, this detection of rapid biomass increase after flood detection in the temporal series is similar to previous paddy mapping with optical time-series data [4]. Any pixel that meets both of these conditions is retained for further processing as a potential rice pixel.

4 Like Rule $2 \mathrm{~b}$, it is also possible that the rice crop was established later than anticipated by the acquisition plan. In this case, agronomic flooding will have been detected late in the time series, and the full temporal signature for rice will not be present. This rule states that, if the duration between the detected $S o S$ date and the last date of the time series (tlast) is less than $t_{\text {minlength }}$, then the pixel is labeled as late rice and excluded from further processing.

5 The final rule looks for any unexpected drops in $\sigma^{\circ}$ between $t=S o S+1$ and $t=t_{\text {maxlength }}$ that would be evidence of either a flood or a new cropping season, depending on the elapsed time between this low-value detection and the $S o S$ detection (compared against $a=$ lowest mean). If this condition is passed, the pixel is labeled as rice. Any pixel that exhibits an unexpected low $\sigma^{\circ}$ value moves to $t=t+1$, and Step 2 is applied again.

Figure 4 shows the temporal signature for selected representative pixels to visualize the resulting classification from applying the rule-based system. Exemplar temporal signatures from the Leyte East site are shown for early rice, rice and late rice and other field crops in the top portion, while water bodies, urban areas, forest and fallow are shown in the lower portion.

The top graph shows that, in the class "rice," the crop shows significant temporal behavior and a large dynamic range ( -15 to $-9 \mathrm{~dB}$ ) during its growth period. This is due to the interaction of microwave radiation with the crop canopy, increasing from the detection of $\sigma^{\circ}$ minimum (field inundation) to the detection of $\sigma^{\circ}$ maximum (around the tillering stage) between acquisitions four and six. In the case of "early rice," the SAR series did not capture the $\sigma^{\circ}$ minimum, since it occurred before the first acquisition; however, an increase in backscatter is observed during the growth period for the first four acquisitions that is typical of the seedling to tillering stage. In the case of "late rice," the first four SAR acquisitions show a high backscatter value because, during that period, the fields were in fallow condition (as confirmed in the ground observations). The backscatter minimum was observed in the sixth acquisition, indicating flooded conditions, followed by an increase in backscatter in the succeeding acquisitions, indicating growth of the rice crop. The other field crop signal shows a distinctly different temporal evolution and the absence of any evidence of a water signal at the start of crop growth.

In the bottom graph, the signatures for water bodies and fallow fields/bare soil show a consistently very low to low $\mathrm{dB}$ value and urban areas as consistently high, whereas areas with consistently dense vegetation over time (for instance, forest, banana plantations, etc.) are characterized by a consistent medium to high backscattering coefficient. Other crops are excluded by the significantly different temporal evolution (in temporal and radiometric terms) of the radar backscattering and the absence of 
a water signal at the start of crop growth. In short, this temporal variation of SAR backscatter differentiates rice fields from other land cover classes [14].

Figure 4. Example temporal signatures from Leyte East in 2013 showing different rice classes (top) and other land uses (bottom) from one season of CSK X-band SAR data, $\mathrm{HH}$ polarization and 46-degree incidence angle. Note the different scale in the vertical axes.

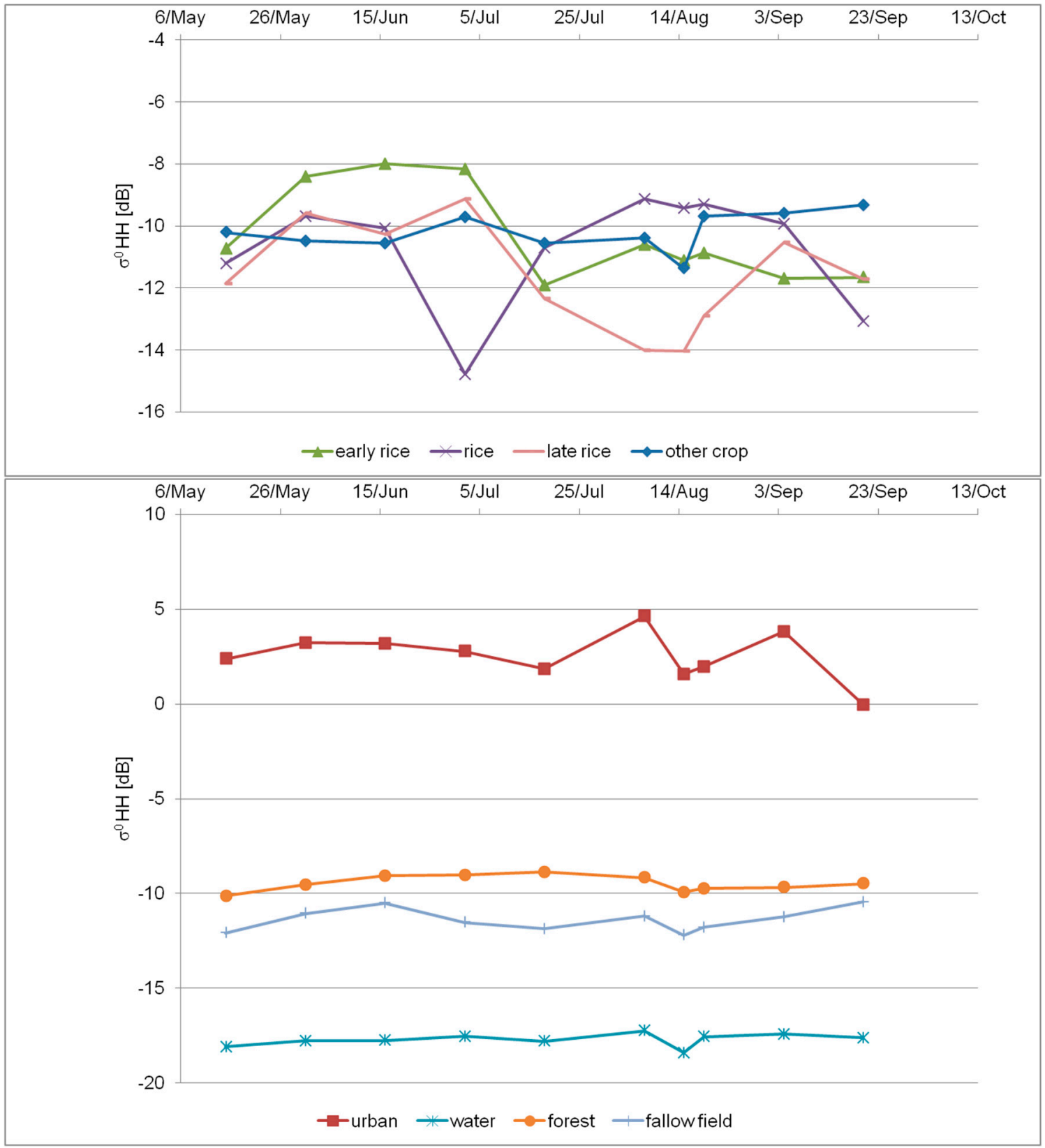

\subsection{Use of Temporal Features to Guide Parameter Selection for the Rule-Based Classifier}

The choice of parameters $a, b, c, d, e$ and $f$ was guided by a simple statistical analysis of the temporal signature of $\sigma^{\circ}$ values in the monitored fields. Table 3 shows the criteria used to guide the selection of parameters. The mean, minimum, maximum and range of $\sigma^{\circ}$ were computed for the temporal signature of each monitored field. Then, we computed the (i) minima and (ii) maxima of those mean $\sigma^{\circ}$ values across fields; the (iii) maxima of the minimum $\sigma^{\circ}$ values across fields; the (iv) minima of the maximum 
$\sigma^{\circ}$ value across fields; and the (v) minimum and (vi) maximum of the range of $\sigma^{\circ}$ values across fields [25]. These six statistics, which we call temporal features, concisely characterize the key information in the rice signatures of the observed fields, and each one relates directly to one parameter. Hence, the value of the six temporal features from the monitoring locations at each site can be used to guide the choice of the six parameter values, as shown in Table 3.

Table 3. Site-specific parameters for the rule-based classification and the criteria used to select them based on temporal features.

\begin{tabular}{ll}
\hline \multicolumn{1}{c}{ Parameter } & Relationship between Parameter and Temporal Feature \\
\hline $\mathrm{a}=$ lowest mean & $\mathrm{a}<$ (i) minima of the mean $\sigma^{\circ}$ across all rice signatures \\
\hline $\mathrm{b}=$ highest mean & $\mathrm{b}>$ (ii) maxima of the mean $\sigma^{\circ}$ across all rice signatures \\
\hline $\mathrm{c}=$ maximum variation & $\mathrm{c}>(\mathrm{vi})$ maxima of the range in $\sigma^{\circ}$ across all rice signatures \\
\hline $\mathrm{d}=$ max value at $S o S$ & $\mathrm{~d}>$ (iii) highest minima in $\sigma^{\circ}$ across all rice signatures \\
\hline $\mathrm{e}=$ min value at peak & $\mathrm{e}<\left(\right.$ iv) lowest maxima in $\sigma^{\circ}$ across all rice signatures \\
\hline $\mathrm{f}=$ minimum variation & $\mathrm{f}<(\mathrm{v})$ minima of the range in $\sigma^{\circ}$ across all rice signatures \\
\hline
\end{tabular}

The parameters $t_{\text {minlength }}, t_{\text {maxlength }}$ and $t_{2}-t_{1}$ are easier to estimate. $t_{\text {minlength }}$ restricts the number of days between a start-of-season detection and the subsequent highest $\sigma^{\circ}$ value in the temporal signature. Since $\mathrm{X}$-band $\sigma^{\circ}$ saturates before rice flowering [15], this value can be set to $40-70$ days. $t_{\text {maxlength }}$ restricts the duration between two $\sigma^{\circ}$ minima in the series and 120 days is a suitable cut-off that would be representative of an intensive triple-rice system (three crops in one year). $t_{2}-t_{1}$ is the maximum duration of agronomic flooding at the start of the season, which can be set to a relatively high value of 40 to 50 to capture even the longest land preparation phases.

\subsection{Rice Map Accuracy Assessment}

A standard confusion matrix was applied to the rice/non-rice validation points collected at each site. The overall accuracy of the rice/non-rice classification and the kappa value were recorded.

\section{Results and Discussion}

The results of the parameter determination and map accuracy assessment are presented and discussed in turn. These are followed by a general discussion section that critiques the methods and suggests ways forward towards a regional-scale rice area mapping and monitoring using this approach.

\subsection{Temporal Features and Parameter Values}

Temporal signatures were extracted for each monitoring field and used to generate the six temporal features. The temporal signatures observed in the monitoring locations for each site are provided in the Supplemental Information section (Table S2). Fields where the rice crop did not reach maturity because of flood or drought were excluded (11 out of 228 were excluded, less than 5\%). The criteria in Table 3 were applied to the temporal feature values and used to select the six parameter values for each site. The temporal features and their related parameters are shown in Table 4, where shaded table cells highlight cases where the relationship between the temporal feature and the parameter (see Table 3 ) was not met. 
$t_{\text {maxlength }}$ was held constant at 120 days; tminlength was held constant at 60 days; and $t_{2}-t_{1}$ was kept constant at a very conservative 45 days across all sites.

Table 4. Summary of the selected parameters (in $\mathrm{dB}$ ) used for the rice classification at each site and the corresponding temporal feature values extracted from the field data. Shaded cells represent cases where the selected parameter did not follow the temporal feature criterion.

\begin{tabular}{|c|c|c|c|c|c|c|c|}
\hline $\begin{array}{l}\text { Site } \\
\text { ID }\end{array}$ & Country, Study Site & a $<$ (i) & b $>$ (ii) & $c>(v i)$ & d $>$ (iii) & e $<$ (iv) & $\mathbf{f}<(\mathbf{v})$ \\
\hline 1 & Cambodia, Takeo & $-18.0<-11.5$ & $-6.0>-10.6$ & $20.0>12.3$ & $-11.0>-14.6$ & $-13.0<-7.3$ & $4.0<7.5$ \\
\hline 2 & Philippines, Leyte East & $-14.0<-12.7$ & $-7.5>-8.3$ & $20.0>8.6$ & $-11.0>-11.8$ & $-10.5<-9.9$ & $3.0<3.3$ \\
\hline 3 & $\begin{array}{l}\text { Philippines, } \\
\text { Leyte West }\end{array}$ & $-14.0<-11.1$ & $-7.5>-7.4$ & $20.0>12.3$ & $-11.0>-12.8$ & $-10.5<-9.1$ & $3.0<3.7$ \\
\hline 4 & $\begin{array}{c}\text { Philippines, } \\
\text { Agusan del Norte }\end{array}$ & $-14.0<-10.6$ & $-7.5>-7.9$ & $20.0>12.5$ & $-8.0>-11.4$ & $-10.5<-9.1$ & $3.0<4.2$ \\
\hline 5 & Vietnam, Soc Trang & $-15.5<-12.0$ & $-7.5>-8.8$ & $20.0>13.3$ & $-10.5>-12.0$ & $-10.0<-9.6$ & $3.0<4.5$ \\
\hline 6 & Vietnam, Nam Dinh & $-15.5<-12.2$ & $-7.5>-8.6$ & $20.0>12.5$ & $-10.5>-12.1$ & $-11.0<-9.6$ & $3.0<4.7$ \\
\hline 7 & Indonesia, Subang & $-17.5<-15.4$ & $-6.0>-11.1$ & $20.0>12.0$ & $-12.0>15.6$ & $-12.0<-11.1$ & $4.0<6.3$ \\
\hline 8 & $\begin{array}{c}\text { India, Tamil Nadu, } \\
\text { Cuddalore }\end{array}$ & $-14.0<-11.9$ & $-8.0>-8.7$ & $20.0>8.2$ & $-12.0>-12.02$ & $-11.0<-9.6$ & $3.0<3.2$ \\
\hline 9 & $\begin{array}{c}\text { India, Tamil Nadu, } \\
\text { Thanjavur }\end{array}$ & $-14.0<-10.1$ & $-8.0>-9.1$ & $20.0>9.6$ & $-11.0>-12.5$ & $-11.0<-7.0$ & $3.0<6.1$ \\
\hline 10 & $\begin{array}{c}\text { India, Tamil Nadu, } \\
\text { Sivaganga }\end{array}$ & $-14.0<-12.1$ & $-9.0>-9.04$ & $20.0>9.3$ & $-12.0>-11.7$ & $-12.0<-9.3$ & $3.0<3.9$ \\
\hline 11 & Thailand, Muang Yang & $-14.0<-11.7$ & $-4.0>-6.2$ & $20.0>9.5$ & $-8.0>-10.4$ & $-10.5<-9.6$ & $3.0<3.6$ \\
\hline 12 & Thailand, Suphan Buri & $-15.5<-13.1$ & $-7.5>-8.6$ & $20.0>11.8$ & $-10.5>-12.0$ & $-10.5<-11.1$ & $3.0<2.11$ \\
\hline 13 & $\begin{array}{c}\text { Philippines, Nueva } \\
\text { Ecija }\end{array}$ & $-14.0<-12.6$ & $-7.5>-8.5$ & $20.0>10.9$ & $-11.0>-10.96$ & $-10.5<-10.4$ & $3.0<3.5$ \\
\hline
\end{tabular}

From the table, it can be noted that the six rice parameters (expressed in $\mathrm{dB}$ ) are consistent across sites and that the choice of parameters agreed with the temporal feature descriptors criteria in all but five out of 78 cases, and in two of those cases, the criteria were very close to being met. We provide some specific comments on the parameters and the additional user knowledge required at some sites to refine the parameter value:

$a$, defined as the lowest mean of the temporal data stack, is meant to mask out the lowest values, typically occurring over water and was set in the range of -18 to $-14 \mathrm{~dB}$. In a few cases, it was clear from visual interpretation of the imagery that this setting was not sufficient. For the three sites in Tamil $\mathrm{Nadu}$, water tanks were not always excluded, because of the cycle of drainage and replenishment for irrigation. In Soc Trang, the draining of aquaculture ponds led to the same effect. In these specific cases, a simple threshold should be augmented with the use of ancillary data to mask out such features.

$b$, defined as the highest mean of the temporal data stack, is the inverse of $a$ and masks out the strong backscatter, which is typical of settlements, and was set in the range of -7.5 to $-9 \mathrm{~dB}$. At only one site was the temporal feature criterion not met, by the small margin of $0.1 \mathrm{~dB}$.

$c$ and $f$ correspond to the maximum and minimum variation, reflecting the range of backscatter within the rice temporal signature over the whole season, hence excluding land cover, such as forest, that has 
temporally-stable backscatter over these durations. For X-band $\mathrm{HH}$ polarization, the highest variation in rice is typically observed between the agronomic flooding and the tillering stage. $c$ was held constant at $20 \mathrm{~dB}$ across all sites as a conservative estimate of the maximum variation, which in practice never exceeded $14 \mathrm{~dB} . f$ was set to a conservative value of 3 or $4 \mathrm{~dB}$, which is at the lower end of the variation expected from a rice crop. At only one site, Suphan Buri, was the temporal feature criterion not met. This site exhibited an extremely complex temporal pattern of rice cropping practices, often in very small fields at the limit of the spatial resolution of the filtered time series.

$d$, defined as the maximum value at $\operatorname{SoS}$ (i.e., at agronomic flooding), is typically expected at around $-18 \mathrm{~dB}$. However, the maximum was set at around $-11 \mathrm{~dB}$ in order to not omit rice fields where the acquisition date was not synchronized with the agronomic flooding. Our observation here is that it is crucial that SAR data, in particular at higher frequencies, such as X-band, be acquired with a high repeat cycle. Moreover, wind conditions, vegetation over the water and field heterogeneity (i.e., part of the fields are not fully covered by water) can cause random scattering, thereby contributing to the backscattering coefficient increase. Finally, land practices or water scarcity also have an impact on the setting of this parameter; the two rainfed sites of Muang Yang and Agusan del Norte are examples where the temporal feature descriptor was correspondingly low. The temporal feature criterion was not met in Sivaganga, where rice is sown in a semi-dry practice and was marginally not met in Nueva Ecija.

$e$, the minimum value at maximum peak, corresponds to the tillering stage at this frequency and polarization (double bounce). $d$ was often higher than $e$. The key reason is, again, the synchronization between acquisition date and the tillering stage, which at this frequency and polarization has a short length. Suphan Buri was the one site where the parameter was set outside the suggested criterion.

In summary, the temporal feature descriptors give bounding values for the selection of the rule-based parameters in most cases. Operator experience, local knowledge and ancillary data will improve the algorithms' ability to detect rice. The descriptors only bound the parameters on one side, and it would be desirable to have another set of descriptors to bound the parameters on the other side to complement and support operator skill and local knowledge.

\subsection{Rice Area Maps}

Figure 5 shows rice area maps derived from multi-temporal X-band SAR imagery for all 13 sites. We combine late rice and early rice into one class and distinguish them from rice in the maps below for discussion purposes. Map accuracy considers any of the three rice subclasses as rice.

Takeo (Figure 5a) is largely covered by rice and clearly demarcated into two rice cropping systems, the recession rice crop in green and the early wet-season crop, which is represented here as early rice in the western portion of the footprint. The long acquisition period missed the start of the early wet-season crop, but fully captured the recession rice crop.

The small irrigated areas of Leyte East (Figure 5b) show a lot of heterogeneity in planting with a mixture of early rice, rice and late rice classes. The exemplar rice signatures in Figure 4a were taken from this site, with start of season dates ranging from early May to mid-August. This high variability could be due to insufficient water availability or insufficient labor for transplanting. Although the reason for the heterogeneity is not clear, a longer-duration acquisition plan would help to capture the full rice-growing season. 
Figure 5. (a) Rice area map for Site 1, Takeo, Cambodia; (b) rice area map for Site 2, Leyte East, Philippines; (c) rice area map for Site 3, Leyte West, Philippines; (d) Rice area map for Site 4, Agusan del Norte, Philippines; (e) rice area map for Site 5, Soc Trang, Vietnam; (f) rice area map for Site 6, Nam Dinh, Vietnam; (g) rice area map for Site 7, Subang, Indonesia; (h) rice area map for Site 8, Cuddalore, India; (i) rice area map for Site 9, Thanjavur, India; (j) rice area map for Site 10, Sivaganga, India; (k) rice area map for Site 11, Muang Yang, Thailand; (I) rice area map for Site 12, Suphan Buri, Thailand; (m) rice area map for Site 13, Nueva Ecija, Philippines.

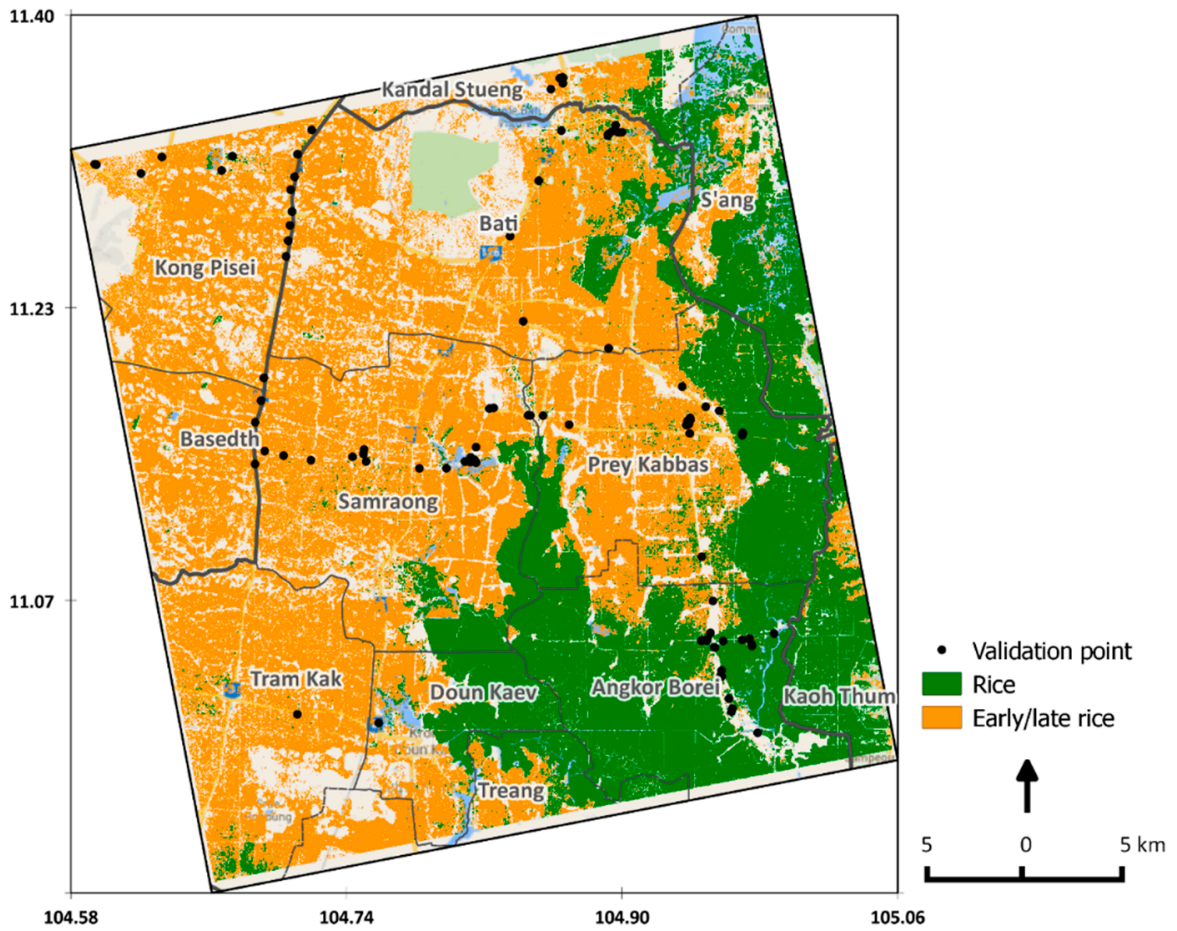

(a)

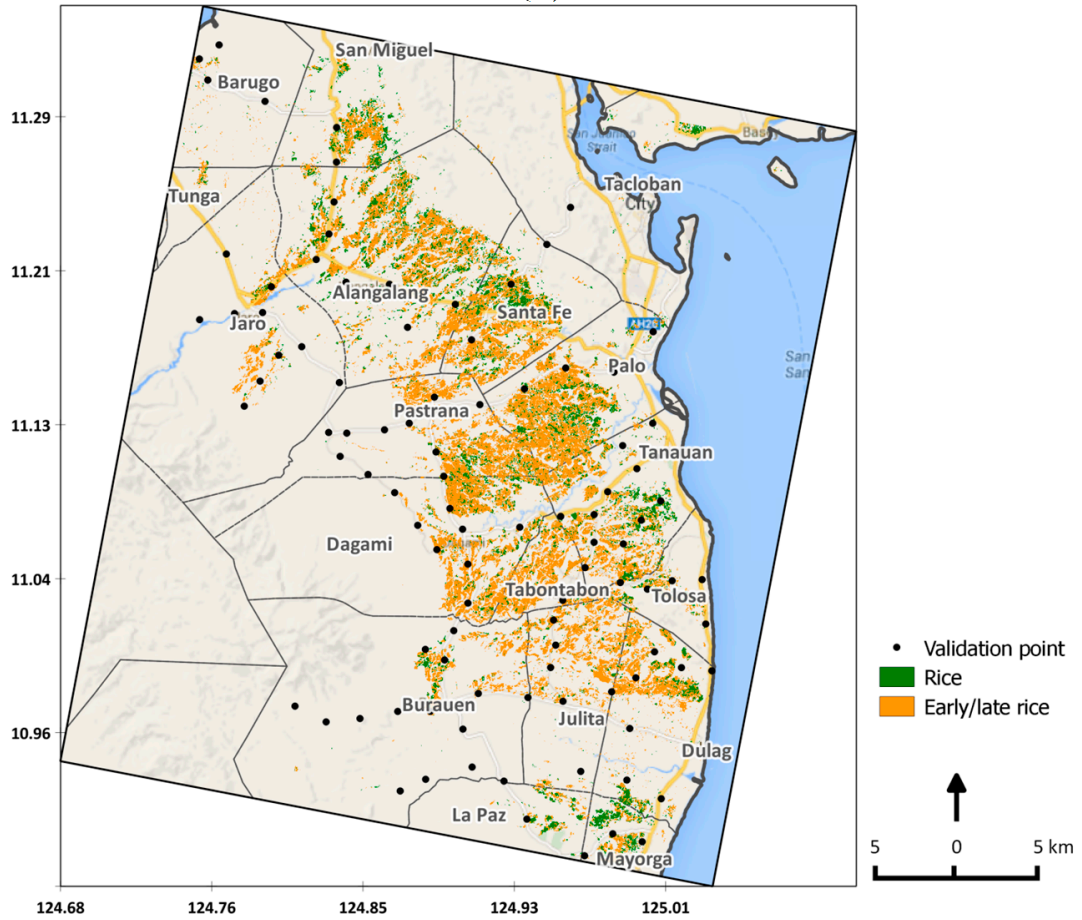

(b) 
Figure 5. Cont.

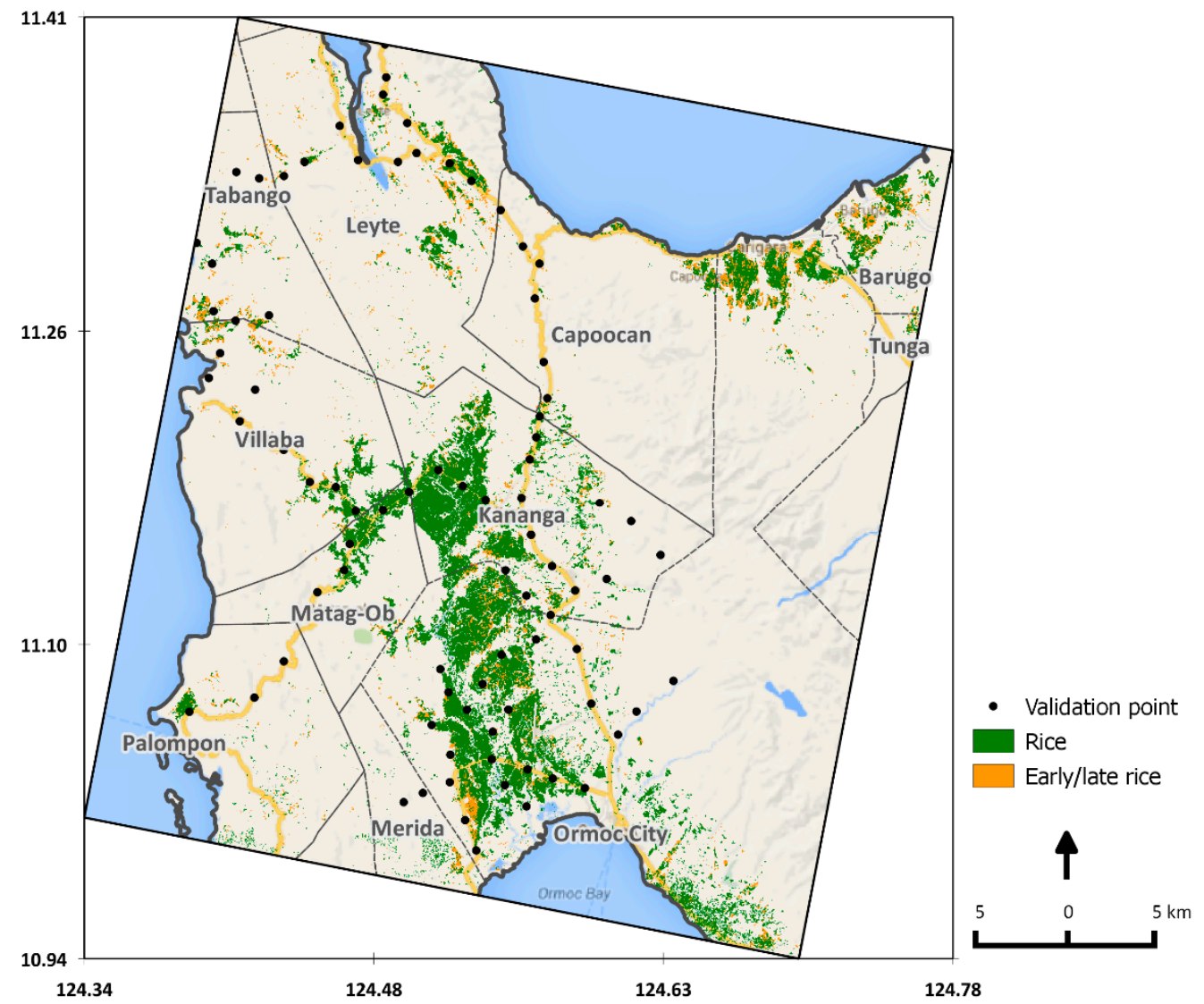

(c)

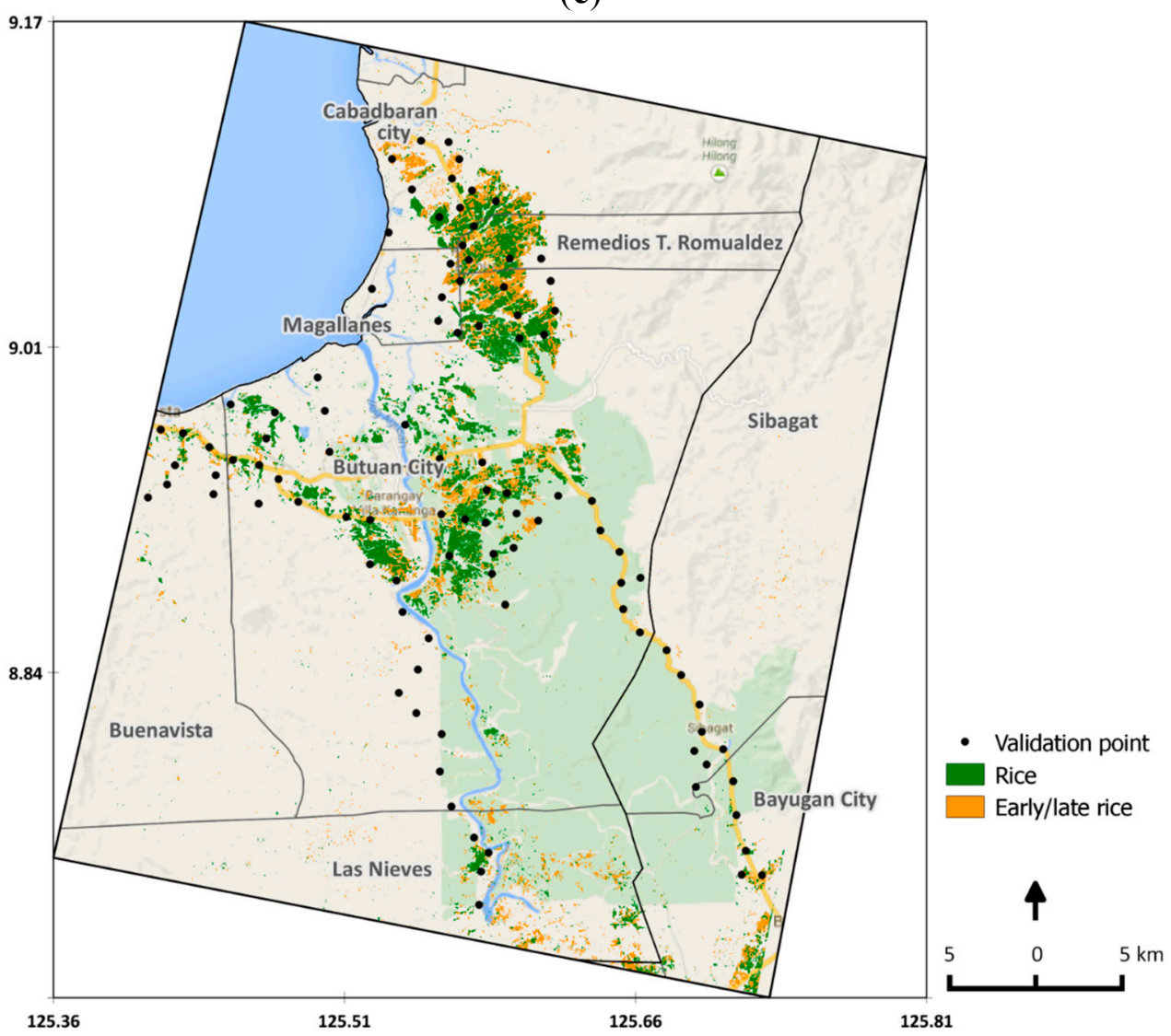

(d) 
Figure 5. Cont.

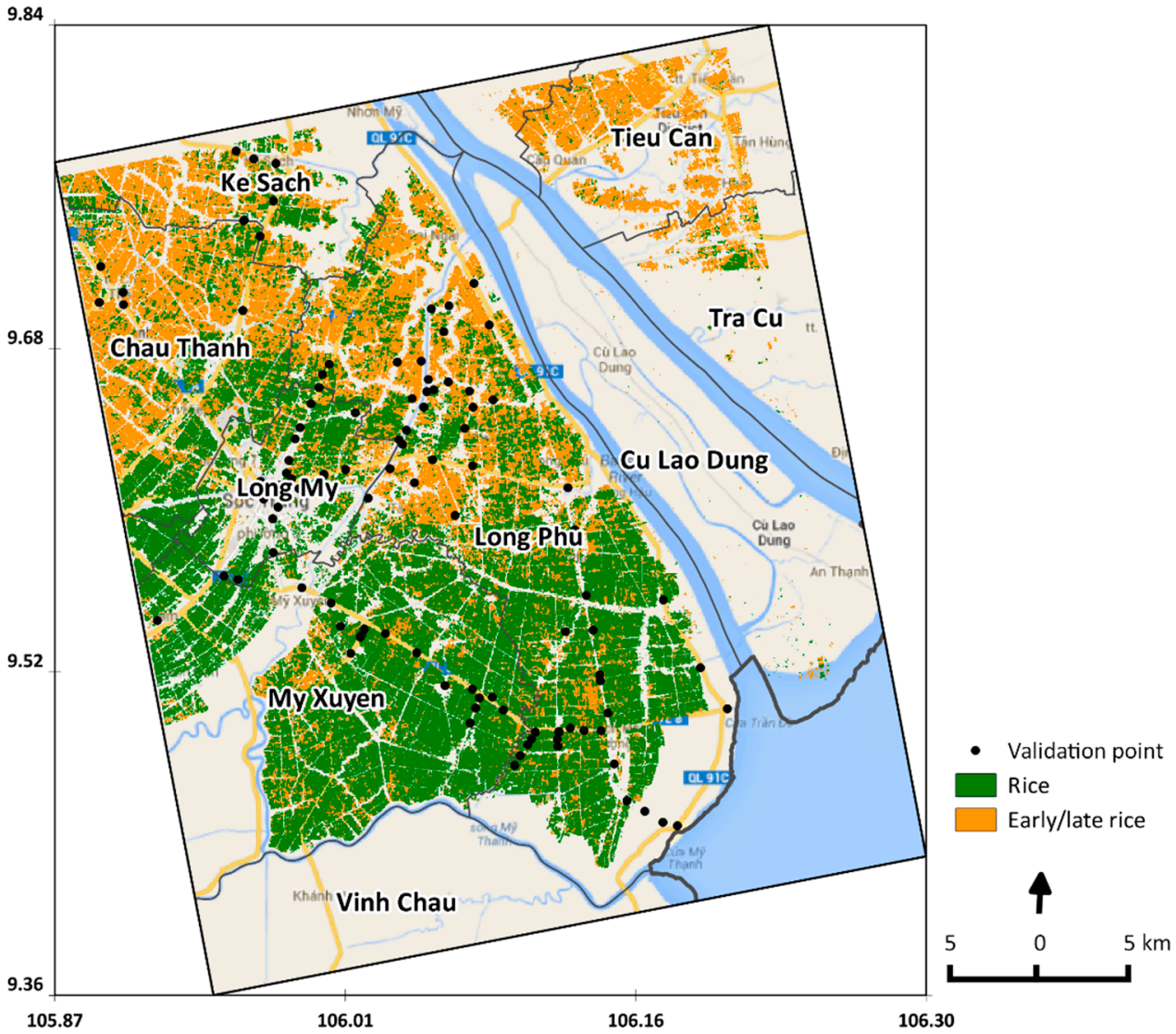

(e)

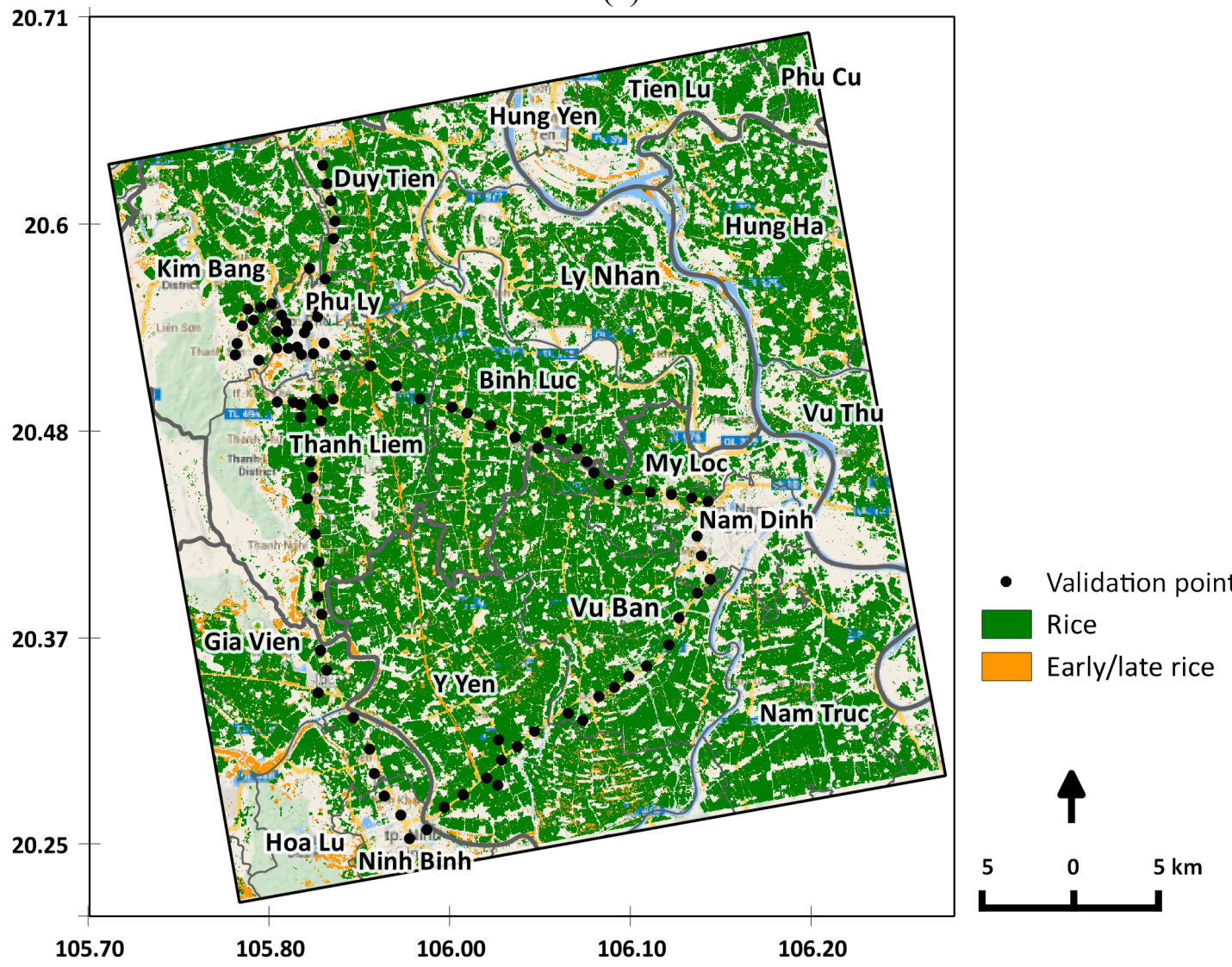

(f) 
Figure 5. Cont.

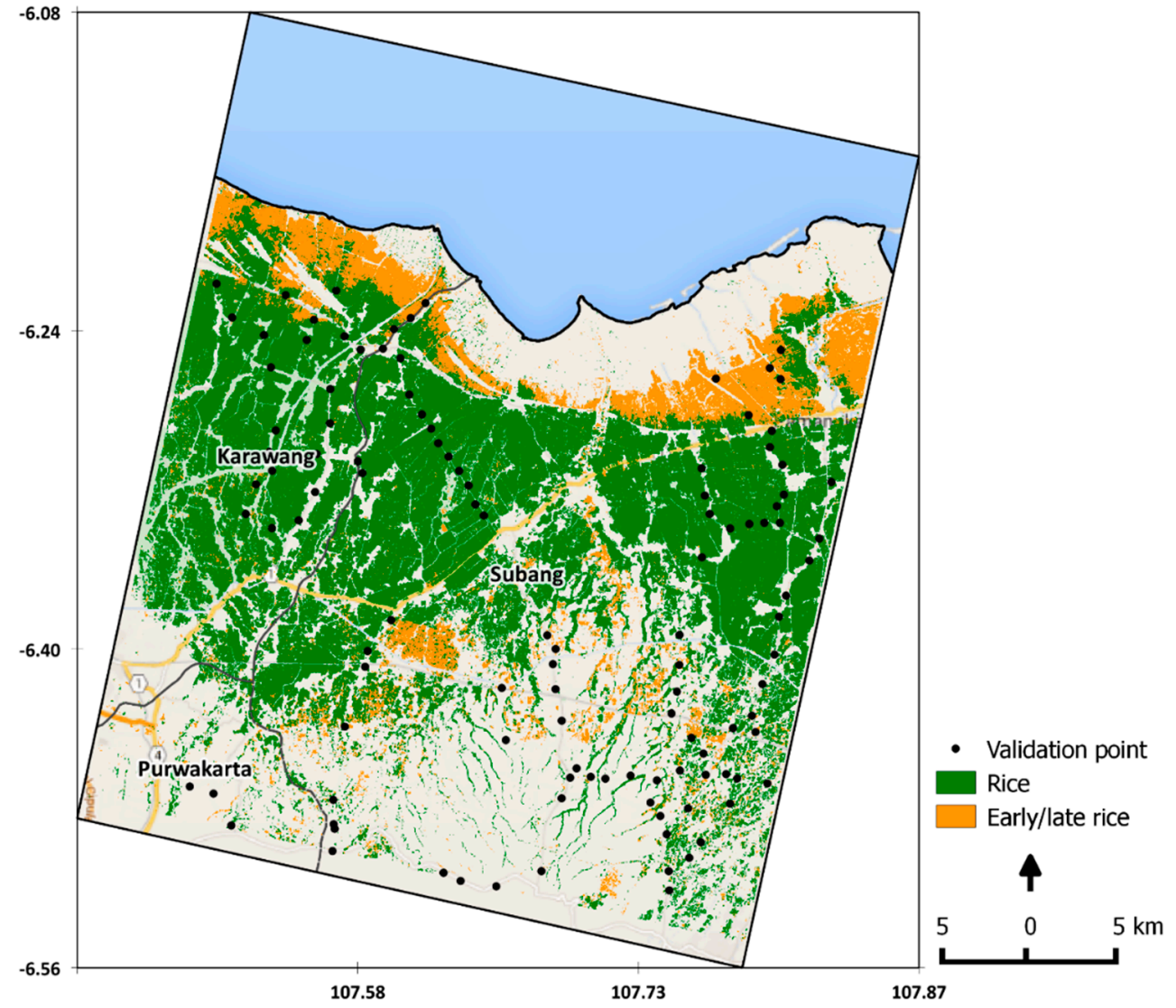

(g)

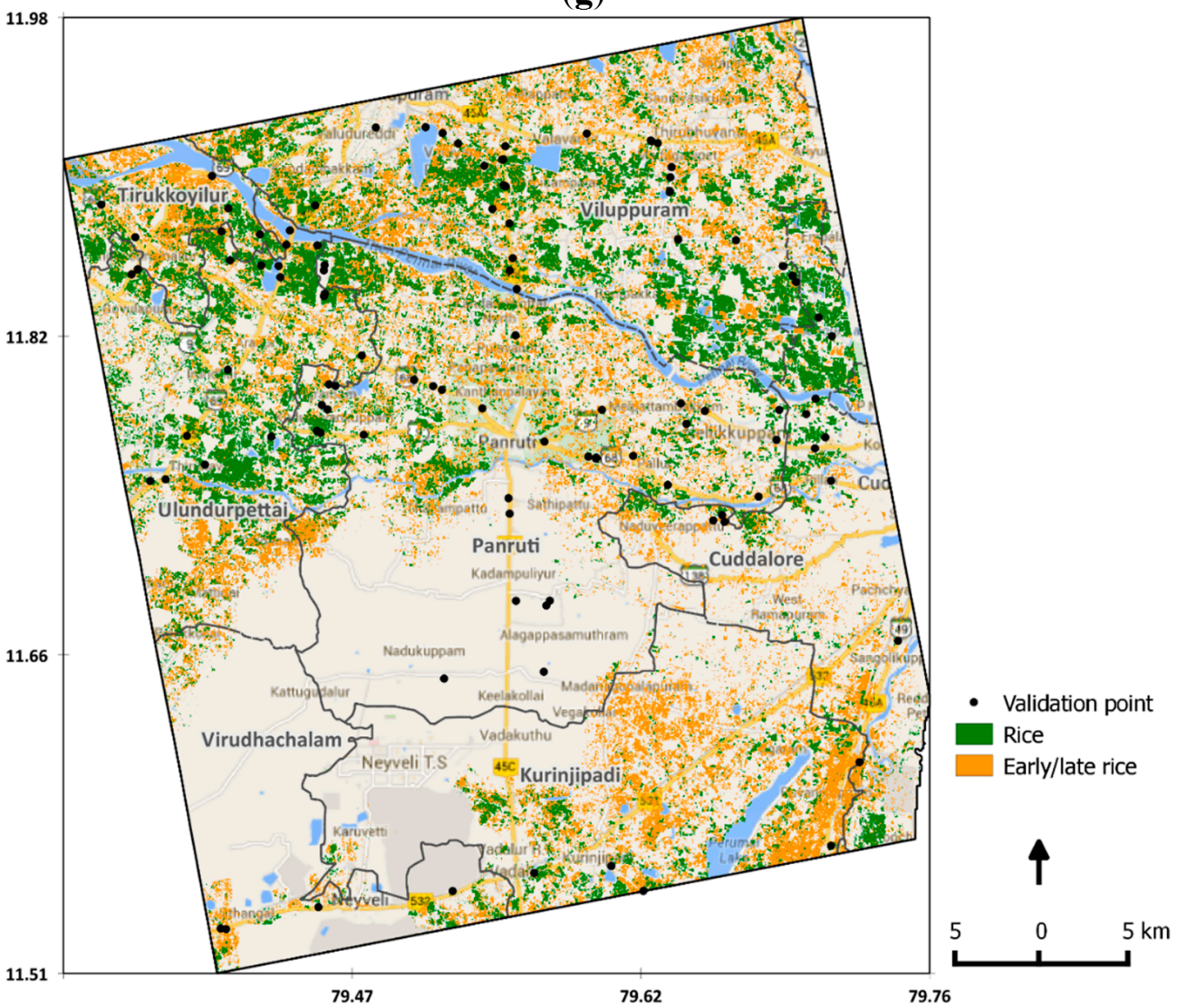

(h) 
Figure 5. Cont.

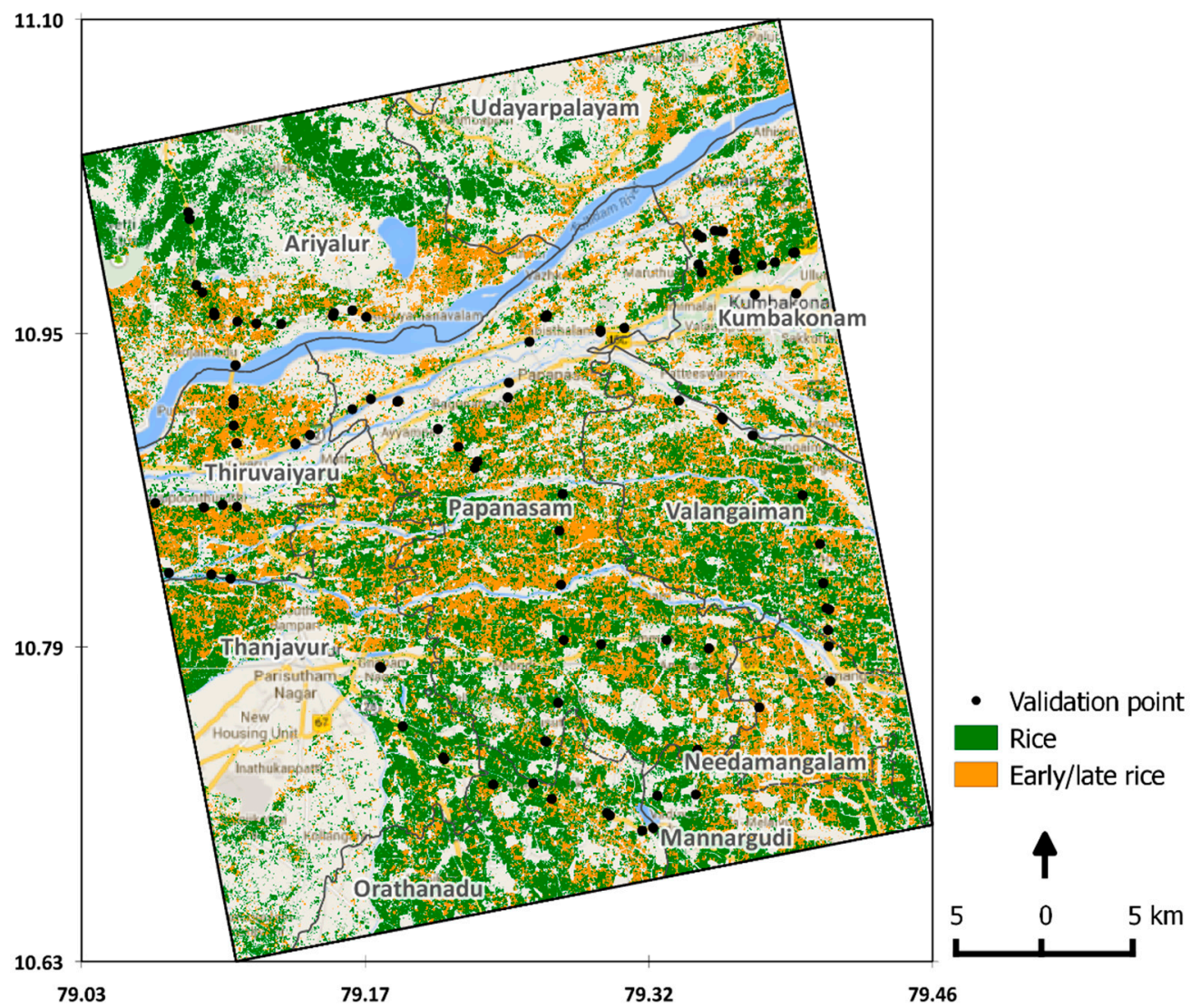

(i)

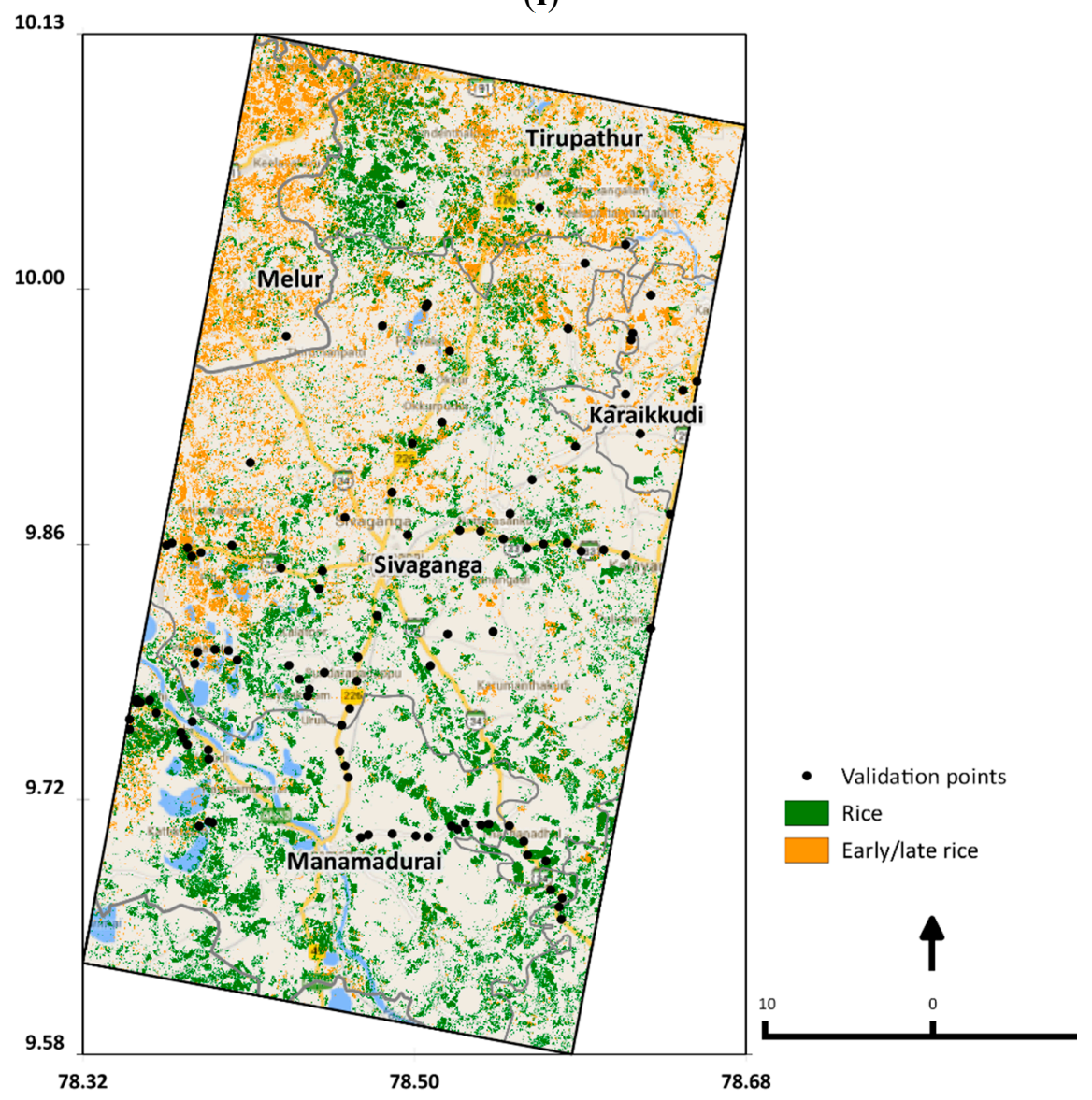

(j) 
Figure 5. Cont.

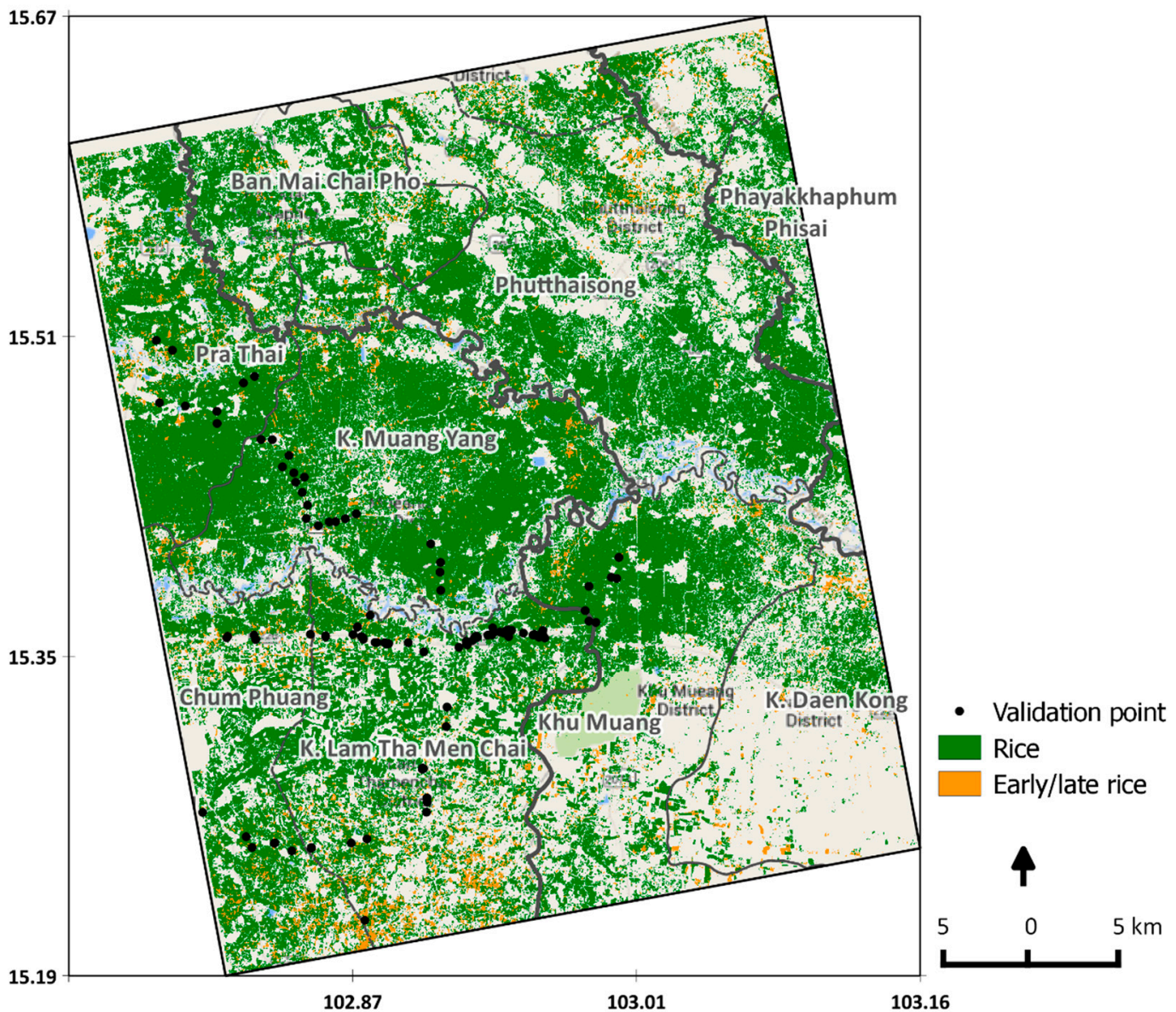

(k)

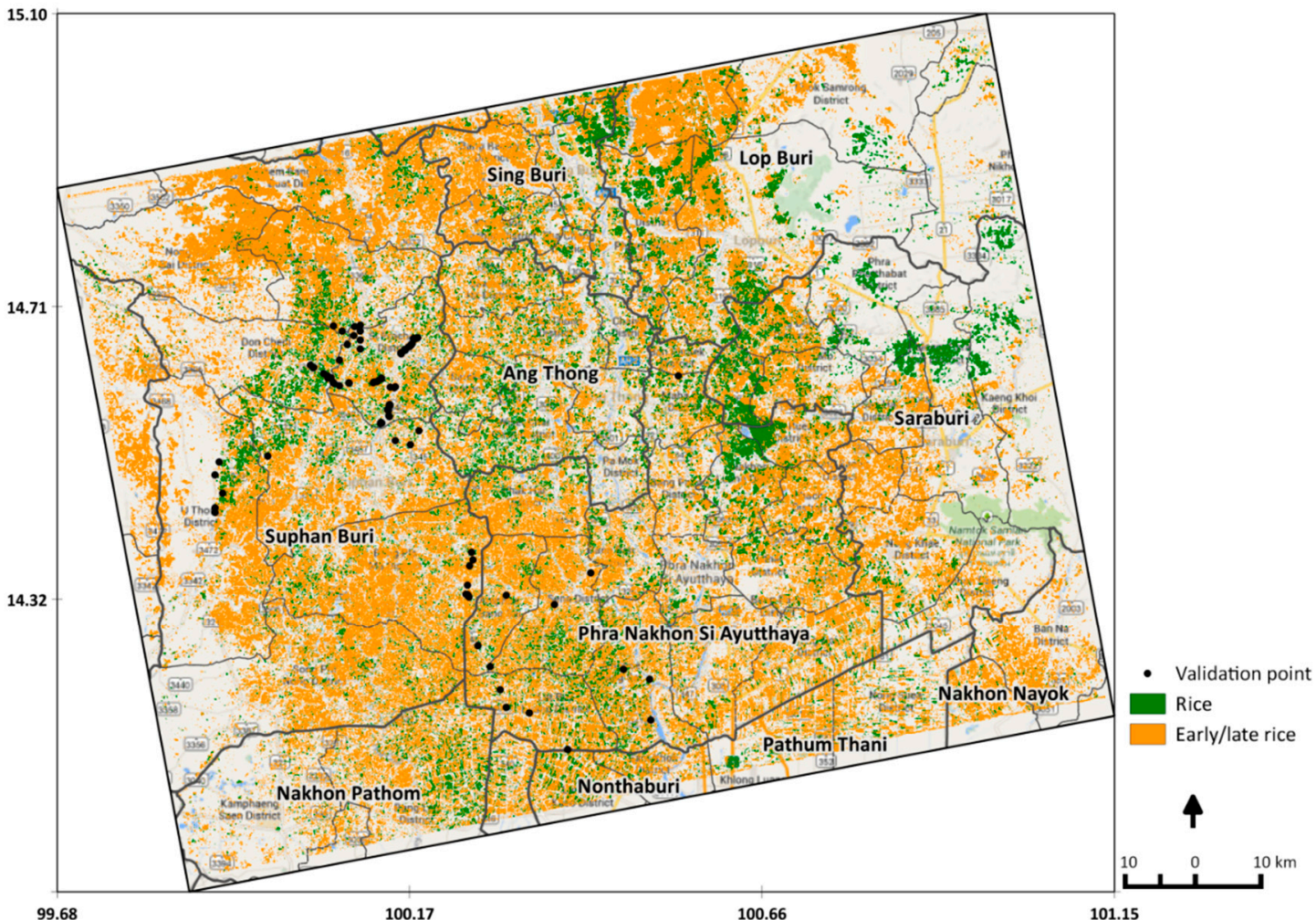

(l) 
Figure 5. Cont.

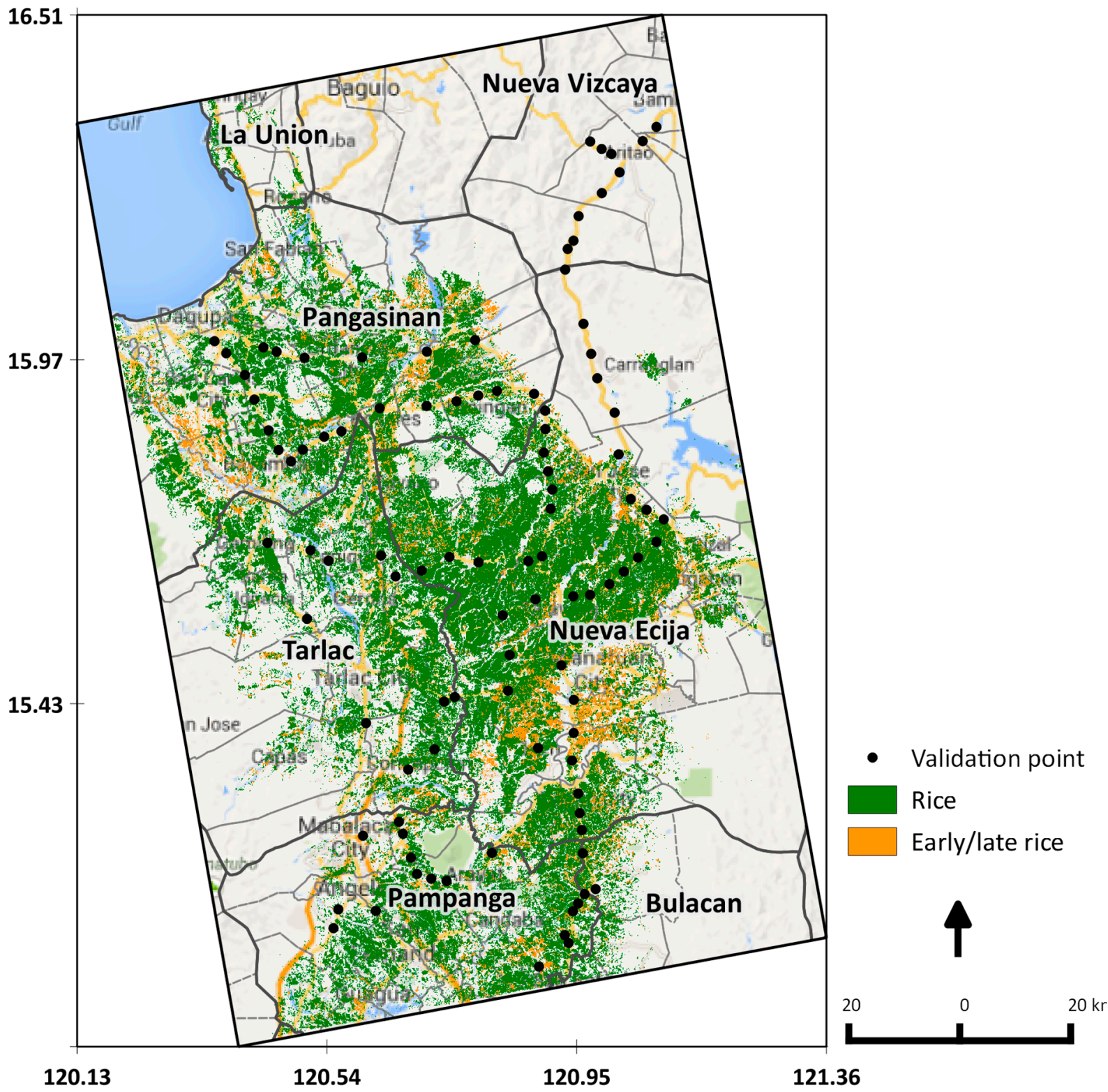

(m)

In contrast to Figure 5b, Leyte West has a much greater homogeneity of rice establishment dates that corresponded well with the acquisition plan.

The main irrigated areas in the northern part of the Agusan del Norte footprint (Figure 5d) are well captured with some apparent difference in planting date on the periphery of the irrigation systems, which could be evidence of insufficient irrigation water in the furthest extents of the system for timely planting. The smaller rainfed areas are in the south.

Soc Trang in the Mekong River Delta (Figure 5e) shows another clear transition from early rice in the northwest to rice in the south. Irrigation canals are clearly visible throughout the rice area.

Nam Dinh (Figure 5f) in the Red River Delta is dominated by rice and, although some canals are visible throughout the rice landscape, they are not as pronounced as in the Mekong; however, settlement and village patterns within the rice area are quite clear. The planting date was extremely regular and corresponded well with the acquisition plan. 
Subang (Figure $5 \mathrm{~g}$ ) is characterized by the release of irrigation water starting in the south and proceeding north to the coast as the season progresses. Since the acquisition period was linked to the first release of irrigation water, it is the coastal areas, planted later, that are classed as late rice, and there are some small areas of early rice in the upper reaches of the system.

Cuddalore (Figure 5h) is another area where the variability in rice crop establishment date is due to the uncertainty in the date of water availability. The clearly demarcated patches in the rice crop in the northern part are water tanks that were successfully excluded from the classification.

Thanjavur (Figure 5i) is a much more intensively cropped area than the other two sites in India, and again, the water tanks that were successfully excluded from the classification are clearly visible as patches in the rice area.

Sivaganga (Figure 5j) was a challenging site to classify because of the sparse rice area and the semi-dry rice crop establishment practice. The irregularity in monsoon rains is one reason for the variation in crop establishment date across the site, resulting in a mixture of early/late rice and rice.

Muang Yang (Figure 5k) is an extensive rainfed system with homogeneous planting dates due to plentiful and timely rainfall in most areas during the monitored season. Field sizes are much larger than those in Suphan Buri, and coarser spatial resolution imagery (in the region of $20 \mathrm{~m}$ ) than used here would be more suitable for rice crop monitoring in the northeast region of Thailand.

Suphan Buri (Figure 51) was another challenging area to map, since the continuous availability of irrigation water and the stable seasonal conditions mean that rice can be established at almost any time, in patterns of double crop, triple crop or five crops in two years. This complexity in crop calendars means that a short-duration acquisition period cannot capture the full rice signature over the entire footprint. Furthermore, the small field sizes at the site were difficult to capture with the coarser spatial resolution used in this large footprint. Longer-duration monitoring or even continuous monitoring with higher spatial resolution would be required to better represent the rice area here.

Nueva Ecija (Figure $5 \mathrm{~m}$ ) is the major rice-growing area of the Philippines, and it relies on water provided by major irrigation facilities - the NIA-UPRIIS (National Irrigation Administration-Upper Pampanga River Integrated Irrigation Systems) service area-for much of the footprint. The water source is the Pantabangan Dam (the reservoir is clearly visible in East of the image), which is one of the largest irrigation facilities in the Philippines. The planting in this season was relatively homogeneous and aligned with the acquisitions, with only a few areas showing early or late planting.

\subsection{Rice Map Accuracy Assessment}

The accuracy assessment is a comparison of the classified rice map against ground-truth data. The spatial resolution of the rice maps ranges from $3 \mathrm{~m}$ to $15 \mathrm{~m}$. However, the ANLD filtering processes (see Section 3.1) reduce the effective resolution by performing locally adaptive smoothing and edge detection. To account for this lower resolution and the horizontal accuracy of the handheld GPS units relative to the pixel size, the validation data were collected in areas that had homogeneous land cover in a 15-m radius around each GPS point for sites using 3-m resolution imagery and a 50-m radius for sites using 10-m or 15-m resolution imagery (see Section 2.2). The observed land cover at the GPS validation points was compared to the mode value of the rice map pixels within a window that matched the radius used in the validation land cover assessment. The accuracy assessment for the rice maps was conducted 
on a rice/non-rice basis, where all other land cover types were grouped into a single non-rice class. Across the 1,334 points, 696 (52.2\%) were rice, 233 (17.5\%) were other annual, perennial or plantation crops (such as banana, coconut, cotton, fruit orchards, cassava, corn, sugarcane, groundnut and black gram), 85 (6.4\%) were built-up areas, $75(5.6 \%)$ were forest, $72(5.4 \%)$ were grassland, 35 (2.6\%) were water bodies, with barren, mixed, shrublands, and others made up the remaining $10.3 \%$.

This rich non-rice dataset (638 points) could be further exploited in the future to assess the SAR signatures of other land cover types commonly found in rice-growing areas. The same signatures could also be used to generate new bounding limits (based on the temporal signatures for other crops and urban and water surfaces, for example) to further guide parameter selection in the rule-based classifier.

Given the large amount of in situ data collection across 13 sites and six countries, we adopted a rapid and practical approach to the sampling method for the map validation (see Section 2.2) as opposed to a regular sampling grid, for example. There is substantial variability in the spatial distribution of the validation points across the sites (Figure 5a-m), which reflects a combination of the following constraints: (i) variability in the level of road access; (ii) the human resources available; (iii) the local teams' familiarity with the landscape; and (iv) availability of ancillary spatial data to guide the team. In many cases (Site 1, Takeo, Site 6, Nam Dinh, Site 8, Cuddalore, Site 9, Thanjavur, Site 10, Sivaganga, Site 11, Muang Yang, Site 12, Suphan Buri, and Site 13, Nueva Ecija), additional validation dates were added to increase the spatial coverage of the points. In total, 37 days of travel and field data collection were required to collect 1334 validation points across the 13 footprints, an average of 36 points per day and 103 points per site (Table 5). It is clear that even this rapid appraisal approach was resource intensive, especially in areas where accessibility was limited.

Table 5. Summary of site validation visits, rice area and accuracy assessments.

\begin{tabular}{|c|c|c|c|c|}
\hline $\begin{array}{l}\text { Site } \\
\text { ID }\end{array}$ & Country and Study Site & Validation Points and Date(s) of Validation & $\begin{array}{l}\text { Rice Area (ha) and as \% of } \\
\text { Footprint }\end{array}$ & $\begin{array}{c}\text { Accuracy and } \\
\text { Kappa }\end{array}$ \\
\hline 1 & Cambodia, Takeo & 100, 8 April, 22 April and 11 September 2013 & $150,026,94 \%$ & $85 \%, 0.70$ \\
\hline 2 & Philippines, Leyte East & 99, 24-26 September 2013 & $17,817,11 \%$ & $87 \%, 0.74$ \\
\hline 3 & Philippines, Leyte West & 85,27 to 28 September 2013 & $15,229,10 \%$ & $89 \%, 0.79$ \\
\hline 4 & Philippines, Agusan del Norte & $100,14-16$ October 2013 & $13,163,8 \%$ & $89 \%, 0.78$ \\
\hline 5 & Vietnam, Soc Trang & 108, 25 September 2013 & $55,216,35 \%$ & $87 \%, 0.74$ \\
\hline 6 & Vietnam, Nam Dinh & 100, 30 August and 5 September 2013 & $108,733,68 \%$ & $89 \%, 0.78$ \\
\hline 7 & Indonesia, Subang & $115,10-13$ February 2014 & $64,533,40 \%$ & $97 \%, 0.93$ \\
\hline 8 & India, Tamil Nadu, Cuddalore & 111, 12 February and 3 March 2014 & $26,015,16 \%$ & $92 \%, 0.84$ \\
\hline 9 & India, Tamil Nadu, Thanjavur & 102, 31 January, 1 February and 7 March 2014 & $83,871,52 \%$ & $91 \%, 0.82$ \\
\hline 10 & India, Tamil Nadu, Sivaganga & 105,14 and 21 February 2014 & $41,825,24 \%$ & $87 \%, 0.73$ \\
\hline 11 & Thailand, Muang Yang & $\begin{array}{l}\text { 109, } 17 \text { October and } 12 \text { December 2013; 12and } \\
\qquad 28 \text { February } 2014\end{array}$ & $91,908,57 \%$ & $86 \%, 0.72$ \\
\hline 12 & Thailand, Suphan Buri & $\begin{array}{l}\text { 100, } 25 \text { September, } 25 \text { October and } 14 \text { December } \\
\qquad 2013 ; 22 \text { January } 2014\end{array}$ & $555,317,40 \%$ & $87 \%, 0.74$ \\
\hline \multirow[t]{2}{*}{13} & Philippines, Nueva Ecija & 100,19 September, 3 and 4 October 2013 & $424,801,27 \%$ & $86 \%, 0.72$ \\
\hline & Points and area (ha) & 1334 & $1,648,454$ & \\
\hline
\end{tabular}




\subsection{Potential Sources of Misclassification}

Although the classifier can properly detect most rice areas, some land cover types can cause misclassifications. Wetland or seasonal water bodies that are subjected to drying followed by sudden vegetation growth can contribute significantly to an increase in commission errors. This behavior is observed for some rivers that drain very quickly and also for water tanks used to store irrigation water (Sivaganga, for example). The temporal signature of this land cover is similar to the typical rice signature, and if the timing of the event corresponds to the known rice crop calendar, then the discrimination of rice is extremely challenging. One solution is to use multi-temporal SAR or optical images acquired in suitable periods, often outside the rice-growing season, to develop such a mask. In some cases, but this depends on the rice environmental conditions, the use of additional polarizations or the combination of different frequencies (for instance, the C- and L-band, as shown in [25]), which may help in the exclusion of some non-rice areas.

Many of the omission errors were associated with a lack of correspondence between the observed rice crop calendar in isolated areas within the footprint and the acquisition period. This is particularly problematic when rice is sown early with respect to the average crop calendar, since the signature does not include the critical land preparation/agronomic flooding, which is the foundation of rice detection. A further problem relates to extremely short-duration varieties, around 90 days, which are transplanted as 15- or 20-day-old seedlings. This means that the remaining vegetative stage in which biomass increases substantially is of very short duration and can be hard to detect, especially if there are cancellations during that time of the season. Photoperiod-sensitive varieties with a long growth duration, such as those cultivated in Muang Yang, are also challenging, and further work is required to determine the most appropriate parameters for those situations.

We have considered only six temporal feature descriptors in this study, but other features could be extracted and related to the rice crop phenology. This could improve the accuracy, but also further guide the parameter selection by providing bounds on both sides of the parameter value.

\subsection{Observations on the Temporal and Spatial Resolution Requirements for SAR Rice Crop Mapping}

Despite good local knowledge and planning, the acquisitions did not always coincide with the rice crop calendar, resulting in significant areas of late or early rice detection. The complexity of cropping calendars, changes in farmer intentions, unexpected delays to the start of the season and delays in the release of irrigation water all play a role in this. Acquisition plans for rice crop monitoring need to account for this uncertainty in rice calendar information. Year-round monitoring may be needed in areas where rice is cultivated several times a year.

Appropriate temporal frequency plays a key role in the accurate detection of rice area and in other rice crop growth information. For short- and medium-duration crops, as grown in nine of the 13 footprints, the season duration is approximately three to four months, which translates into 6-8 CSK images or 9-12 TSX images per season. For these durations, we found that at least 6-7 images are needed to perform a reliable analysis, and this suggests that a 16-day repetition is the minimum requirement for rice crop monitoring using this approach. Higher temporal frequency would of course be better considering the fast 
development of the rice plant and the sensitivity of the X-band to the different crop stages. Future work should explore the derivation of key crop dates and stages from the same series of images.

High spatial resolution is also important and is related to field size, planting homogeneity and landscape fragmentation. In areas characterized by large paddy fields in homogeneous clusters, lower resolution data could be suitable. The Red River Delta and northeast Thailand are good examples of this rice environment. However, in areas, such as Leyte in the Philippines, where most plots are smaller and where neighboring fields can have completely different crop stages, a higher spatial resolution is desirable to capture rice area and other rice crop information.

Regular acquisitions with high spatial and temporal resolution over a large area will require substantial processing capability, such as scalable cloud computing solutions or the use of high-performance computing clusters for parallel processing. The basic processing chain in MAPscape-RICE (see Section 3.1) to convert SLC data into terrain-geocoded $\sigma^{\circ}$ values is already fully automated and highly suitable for parallel processing, but the rice map classification requires operator decisions on appropriate parameters. A library of parameters for different environments and management situations could increase the efficiency of the classification process.

\subsection{Regional-Scale Mapping and Monitoring of Rice Areas in Asia: A Way Forward}

We have demonstrated that rice area can be accurately classified with X-band HH polarization SAR images across multiple environments and management conditions. We have also demonstrated that a large number of scenes covering a large geographic extent can be quickly analyzed using automated processing combined with rule-based parameters that can be derived by the operator based on information gathered from field observations.

SAR-based rice mapping is not new, but the opportunity to develop operational rice monitoring services based on SAR data is new, and there is a great deal of interest and activity in the region. In addition to national-level projects, several consortia are demonstrating the value of rice crop monitoring with SAR across Asia and elsewhere. The Swiss Agency for Development and the Cooperation-funded RIICE project [30] are exploring both public and private sector applications in food security and crop insurance with the aim to build capacity in-country to sustain such services. The Asia-RiCE [31] component of the GEO Global Agricultural Monitoring (GEOGLAM) initiative aims to ensure that rice crop monitoring issues are given suitable priority and attention within the scope of the full GEOGLAM initiative [32], including the development of the observation requirements. Another project conducted by the Asian Development Bank and supported by the Japan Fund for Poverty Reduction aims to improve the quality and timeliness of rice crop area and production estimates and forecasts through SAR data [33]. In Europe, the ERMES (An Earth Observation Model-based Rice Information Service) project aims to develop a prototype of downstream service dedicated to the rice sector based on assimilation of EO and in situ data within crop yield modeling [34]. In short, there is a clear demand and increasing capacity for using SAR data to monitor rice crops on a large scale.

Current and forthcoming SAR systems, such as CSK, TSX, RADARSAT-2, RISAT-1, Sentinel-1 and ALOS-2, offer an unparalleled opportunity to deliver regular and systematic acquisitions of the rice crop in Asia. This could provide information on seasonal rice area, planting dates, crop growth parameters, harvest dates and the impact of flood, drought and wind on the crop. This can be realized 
only if acquisition plans are aligned to the rice crop extent and rice crop calendar across Asia and if acquisitions are frequent enough. Unfortunately, so far, a key challenge is that countrywide systematic acquisitions are still non-existent. In the past three decades, attempts to obtain application-oriented SAR data archives have been carried out by ESA (ERS-Tandem), NASA/JPL (Shuttle Radar Topographic Mission), JAXA (ALOS PALSAR-1) and DLR/EADS Astrium (TanDEM-X) for topographic and forest applications. We hope that space agencies will follow this model for a wider spectrum of land applications and that, in the medium term, common strategies will be pursued.

Field data collection for validation and calibration of remotely sensed data is the most challenging technical aspect of large-scale rice crop monitoring. The development of protocols for rapid and low-cost field assessments is essential if such monitoring systems are to become operational. These protocols could be based on existing field and farmer surveys that are carried out under current reporting by ministries of agriculture or statistics. Furthermore, the use of smart phones or tablet devices to collect field data (such as photos, GPS coordinates, field observations and farmer surveys) and transmit the data via the mobile network or Wi-Fi network to a central database should be explored.

From our experience in this study across six countries and 13 sites, we recommend the following:

(i) A collaborative effort across consortia to provide space agencies with the current best available rice extent data and rice cropping calendar data for Asia, so that the best systematic acquisition strategies can be developed alongside other acquisition needs.

(ii) Uniform coverage of the major rice-growing areas using high spatial resolution $(5 \mathrm{~m}-20 \mathrm{~m})$ SAR imagery with at least bimonthly frequency, with single or dual polarization and with incidence angles between $37^{\circ}$ and $50^{\circ}$. There are good examples of the benefits of regular, consistent, systematic acquisition planning for monitoring at the national and continental scale [35].

(iii) The development of automated processing chains installed on cloud-based or cluster-based hardware solutions to meet the processing and storage requirements of this large amount of data.

(iv) Further research into the use of temporal feature descriptors to classify rice [25].

(v) The development of an open-access library of signatures for rice, and other crops, across multiple environments based on a range of SAR sensors, wavelengths, polarizations and incidence angles. This would complement comparative analyses at the field level [15].

(vi) The evaluation of mobile devices for field data collection.

These recommendations support the regular provision of SAR-based information services, not only for rice, and could emulate the highly successful MODIS project in terms of both application development and scientific advancement in Earth observation.

The unintended consequences of short-term policies and panic responses, such as those that created the rice price crisis of 2008, led to significant food insecurity and poverty for millions across Asia. The provision of regular, unbiased and accurate information on the rice crop would be a significant contribution to policy support and would reduce the risk of similar events happening in the future. Such an endeavor would require coordinated acquisition plans and open data access policies across SAR providers, in-country capacity building for developers and users of the information and investments in research and policy institutes across the region. This is no minor task, but the societal benefits that would accrue from this could be substantial and directly attributable to remote sensing. 


\section{Conclusions}

This research is a contribution to rice mapping and monitoring methods based on multi-temporal SAR data. We presented a new rule-based algorithm, based on agronomically relevant rules and parameters that classified rice area with consistently high accuracy $(>85 \%)$ across different rice environments and crop management practices (wet season and dry season, irrigated and rainfed, direct seeded and transplanted, short- to long-duration varieties). This is the first time that such diversity in rice systems has been assessed with the same mapping approach. The study demonstrates that regularly acquired $\mathrm{X}$-band HH SAR imagery is suitable for rice crop monitoring across the major rice environments of South and Southeast Asia. Future studies should consider a broader range of SAR wavelengths, polarizations and acquisition angles.

The study used the largest number of sites (13), images (127) and field data observations (228 monitored fields, 1922 monitoring visits and 1334 map validation points) of which we are aware. The study demonstrates that this new rice mapping algorithm is robust, that the parameters can be suitably tuned using local knowledge and field observations and that large-scale rice monitoring is feasible. Future studies should consider mobile devices for field data collection to reduce the costs across larger areas. The study presented the concept of agronomically relevant temporal feature dynamics for rice and provided their respective values for X-band HH SAR with acquisition angles between $39^{\circ}$ and $48^{\circ}$. The research suggests that further temporal feature descriptors should be developed to improve the guidance for parameter selection.

One limitation highlighted by the research is the need for ancillary information on land use/land cover to improve the classification in areas where the temporal signature for rice is similar to that of wetlands, water tanks or other areas with comparable temporal dynamics in both water and vegetation cover. Better access to such data and the development of an open-access library of signatures would improve rice mapping with SAR. This study extracted rice area information only from the temporal series, but in the future, the method should be adapted to extract further rice crop parameters from the temporal signature, such as flooding dates, crop establishment dates, crop stage identification and SAR-derived parameters for input to crop growth simulation models, thus providing spatial and temporal information on both rice area and production for food security applications.

\section{Acknowledgments}

This work has been undertaken within the framework of the Remote Sensing-based Information and Insurance for Crops in Emerging economies (RIICE) program, financially supported by the Swiss Agency for Development and Cooperation (SDC). It was also funded by the CGIAR Global Rice Science Partnership (GRiSP) program.

SAR data were provided by ASI/e-GEOS and GISTDA from COSMO-SkyMed and by InfoTerra GmbH from TerraSAR-X. The maps in Figure 5 are overlaid on Google Maps layers, (C) Google, 2014. The boundaries, colors, denominations and other information shown on any map in this work do not imply any judgment on the part of the authors or their institutes concerning the legal status of any territory or the endorsement or acceptance of such boundaries. 
The authors would like to thank the following individuals and institutions for their contribution to the paper: Rosendo Gutierrez, Leo Kris Palao, IRRI GIS Team; Hoang Quang Tung, Huynh Ngoc Dan, Do Thanh Tung, Nguyen Thi Trang, Tran Thi Tam, Seng Vang, Lim Vanndy, Te Kimsokheng, Hem Sovannarith, Udomluk Thammasamisorn, Decha Ubol, Jimmy Loro, Sorasak Khoomboon; Rice Department of Thailand; Tamil Nadu Agricultural University; Department of Agriculture, Philippines; local government units in Leyte, Agusan del Norte and Nueva Ecija, Philippines; and the Center for Rice Research and Development, Sukamandi, Indonesia.

We thank the three anonymous reviewers for their constructive comments to improve early drafts of this manuscript.

\section{Author Contributions}

Andrew Nelson, Tri Setiyono, Emma Quicho, Jeny Raviz, Pongmanee Thongbai, Francesco Holecz, Luca Gatti, Mary Rose O. Mabalay, Wahyunto, Sellaperumal Pazhanivelan, Amornrat Intrman, Vo Quang Minh, Nguyen Huu Quyen, Duong Van Kham, Sarith Hin and Veasna Touch wrote and edited the manuscript. Francesco Holecz, Massimo Barbieri, Francesco Collivignarelli and Luca Gatti developed and refined the MAPscape-RICE software and trained RIICE project partners to use the software. Jenny Ravis, Aileen Maunahan, Mary Rose O. Mabalay, Wahyunto, Sellaperumal Pazhanivelan, Pandian Kannan, Amornrat Intrman, Preesan Rakwatin, Vo Quoc Tuan, Nguyen Huu Quyen, Veasna Touch and Sarith Hin contributed to the processing of SAR data and the generation of rice map products using MAPScape-RICE, as well as the evaluation of rice map accuracy, with consultation and support from Francesco Holecz and Massimo Barbieri. Mary Rose O. Mabalay, Pristine E. Mabalot, Mabel I. Barroga, Alfie P. Bacong, Norlyn T. Detoito, Glorie Belle Berja, Frenciso Varquez, Wahyunto, Dwi Kuntjoro, Sri Murdiyati, Sellaperumal Pazhanivelan, Pandian Kannan, Petchimuthu Christy Nirmala Mary, Elangovan Subramanian, Amornrat Intrman, Thana Setapayak, Sommai Lertna, Vo Quoc Tuan, Trinh Hoang Duong, Nguyen Huu Quyen, Sarith Hin and Veasna Touch conducted rice map field validation work and collected and processed the field data. Andrew Nelson, Tri Setiyono, Arnel Rala, Emma D. Quicho, Jeny Raviz, Prosperidad J. Abonete, Aileen A. Maunahan, Hannah Zarah Bhatti, Lorena Villano, Pongmanee Thongbai, Francesco Holecz, Massimo Barbieri and Francesco Collivignarelli designed the protocols for field monitoring and conducted training on rice map field validation. Eduardo Jimmy P. Quilang, Duong Van Kham, Pongmanee Thongbai, Manoj Yadav, Chharom Chin and Nguyen Hong Ninh oversaw the RIICE project site monitoring and rice map validation activities. Arnel Rala and Cornelia Garcia contributed to the analysis and visualization of rice maps in the paper.

\section{Conflicts of Interest}

The authors declare no conflict of interest.

\section{References}

1. Maclean, J.L.; Hardy, B.; Hettel, G.P. Rice Almanac, 4th ed.; International Rice Research Institute: Los Baños, Philippines, 2013; p. 298. 
2. FAO FAOSTAT. Available online: http: //faostat3.fao.org/faostat-gateway/go/to/home/E (accessed on 1 June 2014).

3. Timmer, C.P. The Changing Role of Rice in Asia's Food Security; ADB Sustainable Development Working Paper Series, No 15; Asian Development Bank (ADB): Manila, Philippines, 2010; p. 18.

4. Xiao, X.; Boles, S.; Frolking, S.; Li, C.; Babu, J.Y.; Salas, W.; Moore, B. Mapping paddy rice agriculture in South and Southeast Asia using multi-temporal MODIS images. Remote Sens. Environ. 2006, 100, 95-113.

5. Gumma, M.K.; Thenkabail, P.S.; Maunahan, A.; Islam, S.; Nelson, A. Mapping seasonal rice cropland extent and area in the high cropping intensity environment of Bangladesh using MODIS $500 \mathrm{~m}$ data for the year 2010. ISPRS J. Photogramm. Remote Sens. 2014, 91, 98-113.

6. Balagtas, J.V.; Bhandari, H.; Cabrera, E.R.; Mohanty, S.; Hossain, M. Did the commodity price spike increase rural poverty? Evidence from a long-run panel in Bangladesh. Agric. Econ. 2014, 45, 303-312.

7. Mittal, A. The 2008 Food Price Crisis: Rethinking Food Security Policies; United Nations: New York, NY, USA, 2009; pp. 1-40.

8. Dawe, D.; Timmer, P.C. Why stable food prices are a good thing: Lessons from stabilizing rice prices in Asia. Glob. Food Sec. 2012, 1, 127-133.

9. Huke, R.E.; Huke, E.H. Rice Area by Type of Culture: South, Southeast, and East Asia, A Revised and Updated Data Base; International Rice Research Institute: Manila, Philippines, 1997; p. 32 .

10. NASA Cloud Fraction. Available online: http: //neo.sci.gsfc.nasa.gov/view.php?datasetId= MODAL2_M_CLD_FR (accessed on 1 June 2014).

11. Pandey, S.; Byerlee, D.; Dawe, D.; Dobermann, A.; Mohanty, S.; Rozelle, S.; Hardy, B. Rice in the Global Economy: Strategic Research and Policy Issues for Food Security; International Rice Research Institute (IRRI): Los Banos, Philippines, 2010; p. 477.

12. Nguyen, T.T.H.; de Bie, C.A.J.M.; Ali, A.; Smaling, E.M.A.; Chu, T.H. Mapping the irrigated rice cropping patterns of the Mekong delta, Vietnam, through hyper-temporal SPOT NDVI image analysis. Int. J. Remote Sens. 2012, 33, 415-434.

13. Boschetti, M.; Nutini, F.; Manfron, G.; Brivio, P.A.; Nelson, A. Comparative analysis of normalised difference spectral indices derived from MODIS for detecting surface water in flooded rice cropping systems. PLoS One 2014, 9, e88741.

14. Le Toan, T.; Ribbes, F.; Wang, L.F.; Floury, N.; Ding, K.H.; Kong, J.A.; Fujita, M.; Kurosu, T. Rice crop mapping and monitoring using ERS-1 data based on experiment and modeling results. IEEE Trans. Geosci. Remote Sens. 1997, 35, 41-56.

15. Inoue, Y.; Kurosu, T.; Maeno, H.; Uratsuka, S.; Kozu, T.; Dabrowska-Zielinska, K.; Qi, J. Season-long daily measurements of multifrequency ( $\mathrm{Ka}, \mathrm{Ku}, \mathrm{X}, \mathrm{C}$, and $\mathrm{L}$ ) and full-polarization backscatter signatures over paddy rice field and their relationship with biological variables. Remote Sens. Environ. 2002, 81, 194-204.

16. Suga, Y.; Konishi, T. Rice crop monitoring using X-, C- and L-band SAR data. Proc. SPIE 2008, 7104, 710410.

17. Bouvet, A.; le Toan, T.; Lam-Dao, N. Monitoring of the rice cropping system in the Mekong delta using ENVISAT/ASAR dual polarization data. IEEE Trans. Geosci. Remote Sens. 2009, $47,517-526$. 
18. Oh, Y.; Hong, S.-Y.; Kim, Y.; Hong, J.-Y.; Kim, Y.-H. Polarimetric backscattering coefficients of flooded rice fields at L- and C-bands: Measurements, modeling, and data analysis. IEEE Trans. Geosci. Remote Sens. 2009, 47, 2714-2721.

19. Kim, Y.H.; Hong, S.Y.; Lee, Y.H. Estimation of paddy rice growth parameters using L-, C-, X-bands polarimetric scatterometer. Korean J. Remote Sens. 2009, 25, 31-44.

20. Lopez-Sanchez, J.M.; Ballester-Berman, J.D.; Hajnsek, I. First results of rice monitoring practices in Spain by means of time series of TerraSAR-X dual-pol images. IEEE J. Sel. Top. Appl. Earth Obs. Remote Sens. 2011, 4, 412-422.

21. Lopez-Sanchez, J.M.; Cloude, S.R.; Ballester-Berman, J.D. Rice phenology monitoring by means of SAR polarimetry at X-band. IEEE Trans. Geosci. Remote Sens. 2012, 50, 2695-2709.

22. Inoue, Y.; Sakaiya, E. Relationship between X-band backscattering coefficients from high-resolution satellite SAR and biophysical variables in paddy rice. Remote Sens. Lett. 2013, 4, 288-295.

23. Inoue, Y.; Sakaiya, E.; Wang, C. Capability of C-band backscattering coefficients from high-resolution satellite SAR sensors to assess biophysical variables in paddy rice. Remote Sens. Environ. 2014, 140, 257-266.

24. Liew, S.C.; Kam, S.P.; Tuong, T.P.; Chen, P.; Minh, V.Q.; Lim, H. Landcover classification over the Mekong river delta using ERS and RADARSAT SAR images. In Proceedings of the 1997 IEEE International Geoscience and Remote Sensing "Remote Sensing-A Scientific Vision for Sustainable Development”' (IGARSS '97), 3-8 August 1997; Volume 4, pp. 2038-2040.

25. Holecz, F.; Barbieri, M.; Collivignarelli, F.; Gatti, L.; Nelson, A.; Setiyono, T.D.; Boschetti, M.; Manfron, G.; Brivio, P.A.; Quilang, J.E.; et al. An operational remote sensing based service for rice production estimation at national scale. In Proceedings of the Living Planet Symposium 2013, Edinburgh, UK, 9-11 September 2013; ESA: Edinburgh, UK, 2013.

26. Yoshida, S. Fundamentals of Rice Crop Science; International Rice Research Institute (IRRI): Los Baños, Philippines, 1981; p. 270 .

27. Tortora, R.D. A note on Sample size estimation for multinomial populations. Am. Stat. 1978, 32, 100-102.

28. De Grandi, G.F.; Leysen, M.; Lee, J.S.; Schuler, D. Radar reflectivity estimation using multiple SAR scenes of the same target: Technique and applications. In Proceedings of the 1997 IEEE International Geoscience and Remote Sensing "Remote Sensing-A Scientific Vision for Sustainable Development" (IGARSS '97), Singapore, 3-8 August 1997; Volume 2, pp. 1047-1050.

29. Aspert, F.; Bach-Cuadra, M.; Cantone, A.; Holecz, F.; Thiran, J.-P. Time-varying segmentation for mapping of land cover changes. In Proceedings of ENVISAT Symposium, Montreux, Switerland, 23-27 April 2007.

30. RIICE. Available online: http://www.riice.org (accessed on 1 September 2014).

31. Asia-RiCE. Available online: http://asia-rice.org (accessed on 1 September 2014).

32. GEOGLAM Crop Monitor. Available online: http://www.geoglam-crop-monitor.org (accessed on 1 September 2014).

33. Innovative Data Collection Methods for Agricultural and Rural Statistics. Available online: http://www.adb.org/projects/46399-001/main (accessed on 1 September 2014).

34. ERMES. Available online: http://www.ermes-fp7space.eu (accessed on 1 September 2014). 
35. Rosenqvist, A.; Shimada, M.; Suzuki, S.; Ohgushi, F.; Tadono, T.; Watanabe, M.; Tsuzuku, K.; Watanabe, T.; Kamijo, S.; Aoki, E. Operational performance of the ALOS global systematic acquisition strategy and observation plans for ALOS-2 PALSAR-2. Remote Sens. Environ. 2014, doi:10.1016/j.rse.2014.04.011.

(C) 2014 by the authors; licensee MDPI, Basel, Switzerland. This article is an open access article distributed under the terms and conditions of the Creative Commons Attribution license (http://creativecommons.org/licenses/by/4.0/). 